T. Higa

Nagoya Math. J.

Vol. 62 (1976), 141-171

\title{
LES PSEUDO-GROUPES INTRANSITIFS ET LE PROBLEME D'EQUIVALENCE
}

\author{
TATSUO HIGA
}

\section{§ Introduction}

Dans les travaux célèbres [1], [2], [3], E. Cartan a fait des recherches sur les pseudo-groupes, sans distinguer entre les deux cas, transitif ou intransitif. Tout d'abord, Cartan a abouti au théorème qu'il a considéré comme la base de la théorie des pseudo-groupes continus, c'est-à-dire le premier théorème fondamental: Tout pseudo-groupe de Lie admet un prolongement holoédrique opérant sur un certain nombre $r$ de variables $x^{i}$ et défini comme l'ensemble des transformations qui laissent invariants

$1^{\circ}$ un certain nombre de fonctions des $x^{i}$;

$2^{\circ} \quad r$ formes de Pfaff $\omega^{i}(x, y, d x)$ linéairement indépendantes par rapport aux différentielles $d x^{i}$ et dont les coefficients peuvent dépendre d'autres variables auxiliaires $y^{j}$; enfin le prolongement considéré a ses équations de définition du premier ordre (voir [5]). Puis, il a introduit l'équation structurale de manière à caractériser le pseudo-groupe.

En ce qui concerne les pseudo-groupes transitifs, la formulation moderne a été faite, par exemple, dans le travail [12]; il se trouve que les espaces annoncés dans le premier théorème fondamental sont considérés comme les espaces qui construisent l'espace fibré principal de repères ; ce système des espaces est ce qu'on appelle "G-structure". En suivant E. Cartan, nous pouvons définir une certaine fonction sur une $G$-structure. Dans le cas où le pseudo-groupe considéré est transitif, cette fonction structurale doit être constante; alors nous pouvons trouver que deux $G$-structures ayant les mêmes fonctions structurales constantes sont localement isomorphes si $G$ vérifie une certaine condition algébrique; la condition d'involution (voir le Théorème 3-1 de [12]).

Il semble que les pseudo-groupes intransitifs soient aussi importants, non seulement pour la théorie des groupes elle-même, mais encore pour

Received February 24, 1976. 
la théorie sur l'intégration des équations différentielles invariantes par les pseudo-groupes (cf. [8]).

Par exemple, si nous nous proposons de préciser les notions "isomorphe holoédrique" et "isomorphe mériédrique" introduites dans la théorie par E. Cartan, il sera nécessaire de donner la solution au problème d'équivalence des groupes intransitifs. En outre, relatif à la classification des pseudo-groupes intransitifs, ou plutôt des algébres de Lie filtrées intrasitives (et simples), il vaudrait mieux que les pseudo-groupes restreints aux fibres d'une fibration $p: M \rightarrow N$ invariante par le pseudo-groupe donné sur $M$ soient localement isomorphes entre eux. Mais c'est aussi le problème d'équivalence (cf. Théorème 3-4).

Le but de ce Mémoire est de généraliser les résultats obtenus dans le cas transitif au cas intransitif. D'abord, nous introduisons dans $\S 1$ des structures géométriques pour les pseudo-groupes intransitifs. Localement nous pouvons les expriquer comme suivant.

Soit $N$ une variété différentiable et soit $G$ une sous-variété de $N \times G L(n, R)$ telle que $a: G \rightarrow N,(x, g) \mapsto x$, soit une fibration. Nous supposons, en outre, que chaque fibre $G_{x}=a^{-1}(x)(x \in N)$ soit un sousgroupe de Lie de $G L(n, R)$. Soient $p: M \rightarrow N$ une fibration, $F(M)$ l'espace fibré principal des repères de $M$ et $\pi: F(M) \rightarrow M$ la projection. Alors un sous-espace fibré $B$ de $\pi: F(M) \rightarrow M$ sera dit une $G$-structure généralisee (ou simplement $G \cdot G$-structure) si, pour tout $x \in N, B_{x}=(p \cdot \pi)^{-1}(x) \cap B$ est un espace fibré principal de base $M_{x}=p^{-1}(x)$ et de groupe structural $G_{x}$ (cf. [13]).

Nous l'interpréterons comme l'espace auquel Cartan a abouti dans son premier théorème fondamental, et nous considérerons les pseudogroupes (intransitifs) qui laissent invariants les $G \cdot G$-structures. En suite, nous indiquons le théorème qui ramène le probème d'équivalence des pseudo-groupes au problème des $G \cdot G$-structures (Théorème 1-2).

Dans $\S 2$, nous construisons, de plus, la structure géométrique ordinaire $P(B)$ déterminée par une $G \cdot G$-structure $B$. Pour cela, il faut imposer certaine condition aux $G \cdot G$-structures. Alors, sous cette condition, nous pouvons démontrer qu'un isomorphisme local de $P(B)$ dans $P\left(B^{\prime}\right)$ entraîne celui de $B$ dans $B^{\prime}$ et réciproquement (Théorème 2-1). Il en résulte que le normalisateur du pseudo-groupe attaché à $B$ est transitif si $P(B)$ est transitif. Finalement nous introduisons une fonction structurale pour chaque $G \cdot G$-structure satisfaisant à la condition 
ci-dessus.

Dans $\S 3$, tous les objets seront supposés analytiques. Dans ce caslà, en utilisant la théorie des systèmes différentiels extérieurs, nous pouvons démontrer le théorème:

Soient $B, B^{\prime}$ deux $G \cdot G$-structures vérifiant la codition ci-dessus et (s) l'algébre de Lie attachée aux $G \cdot G$-structures $B$ et $B^{\prime}$. Si les conditions suivantes sont vérifiées;

i) (3) est involutif (cf. Définition 3-1),

ii) les deux fonctions structurales $C_{B}$ et $C_{B^{\prime}}$ sont constantes et $C_{B}$ $=C_{B^{\prime}}$,

alors $B$ et $B^{\prime}$ sont localement isomorphes (Théorème 3-3). En particulier, sous la même condition, nous avons le Théorème 3-4: Le normalisateur $\mathrm{du}$ pseudo-grupe déterminé par $B$ est transitif.

Dans $\S 4$, nous allons calculer un exemple très simple.

Dans toute la suite, $\boldsymbol{R}^{n}$ désigne l'espace Euclidien à $n$ dimensions. Le mot "différentiable" signifie à $\S 3$ près "différentiable de classe $C^{\infty}$ ". Soit $M$ une variété différentiable; nous désignons par $T_{x}(M)$ l'espace vectoriel tangent à $M$ en $x \in M$. Soit $f$ une application différentiable d'une variété différentiable $U_{f}$ dans une autre $V_{f} ; f_{*}$ désigne l'application tangente et $f^{*}$ l'application cotangente.

Nous voulons exprimer ici notre profonde reconnaissance aux professeurs M. Kuranishi, A. Morimoto et N. Tanaka qui nous ont donné les séminaires sur ce sujet et les conseils bienveillants.

\section{§1. G-structures généralisées}

Soient $N$ une variété différentiable, $\mathscr{V}$ un fibré vectoriel réel, de base $N$, et $q: \mathscr{V} \rightarrow N$ la projection. Soit $G L(\mathscr{V})_{x}$ le groupe des automorphismes linéaires de la fibre $\mathscr{V}_{x}$ en $x \in N$. Posons $G L(\mathscr{V})=$ $\cup_{x \in N} G L(\mathscr{V})_{x}$, et soit $\pi: G L(\mathscr{V}) \rightarrow N$ l'application canonique. En prenant un sous-ensemble ouvert $U$ de $N$ tel que le fibré vectoriel induit $\left.\mathscr{V}\right|_{U}$ soit trivial, et un isomorphisme de fibrés vectoriels $\alpha$ de $\left.\mathscr{V}\right|_{U}$ sur $U \times \boldsymbol{R}^{n}(n$ est la dimension des fibres de $\mathscr{V}$ ), on peut construire une application bijective $\varphi_{\alpha}$ de $\pi^{-1}(U)$ sur $U \times G L\left(R^{n}\right)$;

$$
\varphi_{\alpha}(u)=\left(x,\left(\left.\alpha\right|_{x}\right) \cdot u \cdot\left(\left.\alpha\right|_{x}\right)^{-1}\right) \quad\left(u \in \pi^{-1}(U), x=\pi(u)\right),
$$

où $\left.\alpha\right|_{x}$ désigne la restriction de $\alpha$ à la fibre $\mathscr{V}_{x}$. Alors il est immédiat de démontrer la proposition suivante. 
Proposition 1-1. L'ensemble $G L(\mathscr{V})$ admet une structure canonique de variété différentiable satisfaisant aux conditions suivantes;

(a) $\pi: G L(\mathscr{V}) \rightarrow N$ est une submersion,

(b) l'application $G L(\mathscr{V}) \ni g \mapsto g^{-1} \in G L(\mathscr{V})$ est différentiable,

(c) l'application $G L(\mathscr{V}) \times_{N} G L(\mathscr{V}) \ni(g, h) \mapsto g \cdot h \in G L(\mathscr{V})$ est différentiable, où $G L(\mathscr{V}) \times_{N} G L(\mathscr{V})$ désigne le fibré produit de $\pi: G L(\mathscr{V}) \rightarrow N$,

(d) l'application bijective, définie par un isomorphisme $\alpha$ au-dessus de $U, \varphi_{\alpha}: \pi^{-1}(U) \rightarrow U \times G L\left(\boldsymbol{R}^{n}\right)$ est un difféomorphisme.

Soient $G$ une variété différentiable, fibrée sur $N$, et $\pi: G \rightarrow N$ la projection. Supposons que chaque fibre $G_{x}=\pi^{-1}(x)(x \in N)$ ait une structure de groupe.

Definition 1-1. Nous dirons que $G$ est un groupe de Lie fibré sur $N$, si et seulement si $G$, muni de cette structure de groupe, satisfait aux conditions (b) et (c) de la Proposition 1-1.

Soit $G^{\prime}$ un sous-ensemble de $G$. Supposons que $G^{\prime}$ soit aussi un groupe de Lie fibré sur $N$ par rapport à la restriction $\left.\pi\right|_{G^{\prime}}$.

DEFINITION 1-2. Nous dirons que $G^{\prime}$ est un sous-groupe de Lie fibré de $G$, si et seulement si les conditions suivantes sont vérifiées ;

(1) chaque fibre $G_{x}^{\prime}=G^{\prime} \cap G_{x}$ est un sous-groupe de $G_{x}$,

(2) $G^{\prime}$ est une sous-variété différentiable de $G$.

Soit $G$ un groupe de Lie fibré sur $N$. D'après la définition, chaque fibre $G_{x}(x \in N)$ est un groupe de Lie ayant la même dimension; mais, en général, les groupes des fibres ne sont pas toujours isomorphes. Cependant, la plupart des exemples pratiques, par exemple, ceux de E. Cartan [2], montreront que des groupes des fibres d'un sous-groupe de Lie fibré de $G L(\mathscr{V})$ sont isomorphes entre eux.

Remarque. En désignant par $G_{0}$ un groupe de Lie, on vérifie aisément que $G=N \times G_{0}$ est un groupe de Lie fibré sur $N$ par rapport à la projection canonique $\pi: N \times G_{0} \rightarrow N$. Lorsque le fibré vectoriel est trivial, i.e., $\mathscr{V}=N \times \boldsymbol{R}^{n}, G L(\mathscr{V})$ s'identifie avec $N \times G L\left(\boldsymbol{R}^{n}\right)$.

Considérons, de plus, une variété différentiable $M$ fibré sur $N$, et soit $\alpha: M \rightarrow N$ la submersion. Dans toute la suite, nous ne considérons qu'une variété $M$ telle que, pour tout $\xi \in N, \operatorname{dim} M=\operatorname{dim} \mathscr{V}_{\xi}$ soit vérifié.

Cela étant, désignons par $F=F(M, \alpha, N, \mathscr{V})$ l'ensemble de toutes les applications linéaires isomorphes de la forme suivante; 


$$
z: \mathscr{V}_{\xi} \rightarrow T_{x}(M) \quad(x \in M, \xi \in N \text { avec } \alpha(x)=\xi),
$$

et définissons une application surjective $\rho$ de $F$ sur $M$ en faisant correspondre à l'élément $z$ ci-dessus l'élément $\rho(z)=x \in M$. Soit $F \times{ }_{N} G L(\mathscr{V})$ le fibré produit de $\alpha \cdot \rho: F \rightarrow N$ et $\pi: G L(\mathscr{V}) \rightarrow N$; alors on peut définir une application $\Phi: F \times{ }_{N} G L(\mathscr{V}) \rightarrow F$, c'est-à-dire une opération de $G L(\mathscr{V})$ sur $F$, par la composition de deux applications linéaires;

$$
\Phi(z, g): \mathscr{V}_{\xi} \stackrel{g}{\longrightarrow} \mathscr{V}_{\xi} \stackrel{z}{\longrightarrow} T_{x}(M)
$$

$\left((z, g) \in F \times{ }_{N} G L(\mathscr{V}), x=\rho(z), \xi=\alpha(x)\right)$.

Soient $U, V$ des sous-ensembles ouverts de $M$ et de $N$, respectivement, tels que $\alpha(U)=V$ et que $\left.T(M)\right|_{U}$ et $\left.\mathscr{V}\right|_{V}$ soient trivials. Prenons un isomorphisme différentiable de fibrés vectoriels $\gamma$ de $\left.T(M)\right|_{U}$ sur $U \times \boldsymbol{R}^{n}$ et celui $\delta$ de $\left.\mathscr{V}\right|_{V}$ sur $V \times \boldsymbol{R}^{n}$, et posons

$$
\sigma(\gamma, \delta)(x)=\left(\left.\gamma\right|_{x}\right)^{-1} \cdot\left(\left.\delta\right|_{\xi}\right): \mathscr{V}_{\xi} \rightarrow T_{x}(M) \quad(x \in U, \xi=\alpha(x)),
$$

où $\left.\gamma\right|_{x}$ (resp. $\left.\left.\delta\right|_{\xi}\right)$ désigne la restriction de $\gamma$ à $T_{x}(M)$ (resp. $\delta$ à $\mathscr{V}_{\xi}$ ). $\sigma(\gamma, \delta)$ est une section de $\rho: F \rightarrow M$ définie sur $U$.

Proposition 1-2. L'ensemble $F=F(M, \alpha, N, \mathscr{V})$ admet une structure canonique de variété différentiable telle que

(1) $\rho: F \rightarrow M$ est une submersion,

(2) l'opération $\Phi$ de $G L(\mathscr{V})$ sur $F$ est différentiable,

(3) la section $\sigma(\gamma, \delta)$, définie par les isomorphismes $\gamma$ et $\delta$, est différentiable.

Démonstration: Pour la section $\sigma(\gamma, \delta)$ définie ci-dessus, on peut définir une application bijective $\phi_{r, \delta}$ du fibré produit $U \times{ }_{N} G L(\mathscr{V})$ sur $\rho^{-1}(U)$;

$$
\phi_{r, \delta}(x, g)=\sigma(\gamma, \delta)(x) \cdot g, \quad(x, g) \in U \times_{N} G L(\mathscr{V}) .
$$

Donc on sait que $F$ admet une structure de variété différentiable de manière que chaque ensemble $\rho^{-1}(U)$ soit un sous-ensemble ouvert de $F$ et que chaque application bijective $\phi_{r, \delta}$ soit un difféomorphisme. Il est immédiat de constater que $F$, muni de cette structure, satisfait aux propriétés (1), (2) et (3).

Lorsque $N$ a un seul élément, $\mathscr{V}$ peut être considéré comme $\boldsymbol{R}^{n}$ $(n=\operatorname{dim} M)$ et $G L(\mathscr{V})$ comme $G L\left(\boldsymbol{R}^{n}\right)$. Donc, dans ce cas-là, il en resulte 
que $F$ n'est pas autre chose que le fibré de repères de $M$. La proposition suivante sera aussi évidente.

Proposition 1-3. Si $\mathscr{V}$ est trivial, i.e., $\mathscr{V}=N \times \boldsymbol{R}^{n}, F(M, \alpha, N, \mathscr{V})$ s'identifie à l'espace fibré principal de repères de $M$.

Soient $G$ un sous-groupe de Lie fibré de $G L(\mathscr{V})$ et $B$ une sous-variété différentiable de $F(M, \alpha, N, \mathscr{V})$.

Definition 1-3. Nous dirons que $B$ est une G-structure généralisée (ou simplement $G \cdot G$-structure) de groupe structural $G$ et de base ( $M, \alpha$, $N, \mathscr{V})$, si et seulement si les conditions suivantes sont vérifiées;

i) $\rho(B)=M$,

ii) pour tous $z \in B, g \in G$ tels que $z \cdot g$ soit défini, i.e., $(z, g) \in B \times{ }_{N} G$, $z \cdot g \in B$ est vérifié,

iii) pour tous $z, z^{\prime} \in B$ avec $\rho(z)=\rho\left(z^{\prime}\right)$, il existe un élément $g \in G$ tel que $z^{\prime}=z \cdot g$,

iv) pour tout $x \in M$, il existe un voisinage ouvert $U$ de $x$ et une application différentiable $\sigma$ de $U$ dans $B$ telle que $\rho \cdot \sigma=\mathrm{id}_{U}$.

Lorsque $N$ a un seul élément, $B$ est ce qu'on appelle une $G$-structure. Si $\mathscr{V}=N \times \boldsymbol{R}^{n}$, une $G \cdot G$-structure est une sous-variété de l'espace fibré des repères de $M$, vérifiant les conditions précédantes. On a aussi

$$
\operatorname{dim} B=\operatorname{dim} M+\operatorname{dim} G-\operatorname{dim} N,
$$

pour toute $G \cdot G$-structure.

Soit $B(M, \alpha, N, \mathscr{V})$ une $G \cdot G$-structure de groupe structural $G$. Définissons une forme différentielle extérieure de degré $1, \theta$, à valeurs dans le fibré vectoriel $\mathscr{V}$;

$$
\theta_{z}(X)=z^{-1}\left(\rho_{*} X\right) \quad\left(z \in B, X \in T_{z}(B)\right) .
$$

$\theta$ sera dit la forme extérieure canonique sur $B$. Si $\mathscr{V}=N \times \boldsymbol{R}^{n}, \theta$ est considéré comme une forme à valeurs dans $\boldsymbol{R}^{n}$ et on peut définir sa différentielle extérieure $d \theta$.

Maintenant nous allons développer la méthode pour obtenir des $G \cdot G$-structures. $\quad M$ étant une variété différentiable, désignons par $G P(M)$ le pseudo-groupe de toutes les transformations locales de $M$ dans $M$, et par $\pi^{1}(M)$ l'ensemble des jets inversibles d'ordre 1 de $M$ dans $M$, i.e., 


$$
\pi^{1}(M)=\left\{j_{x}^{1}(f) \in J^{1}(M, M) \mid f \in G P(M), x \in U_{f}\right\},
$$

où $J^{1}(M, M)$ désigne la variété des jets d'ordre 1 de $M$ dans $M . \pi^{1}(M)$ est une sous-variété différentiable de $J^{1}(M, M)$. L'application $a: \pi^{1}(M) \rightarrow$ $M, a\left(j_{x}^{1}(f)\right)=x\left(\right.$ resp. $\left.b: \pi^{1}(M) \rightarrow M, b\left(j_{x}^{1}(f)\right)=f(x)\right)$, est appelée l'application source (resp. but). Pour tous $z, z^{\prime} \in \pi^{1}(M)$ tels que $z=j_{x}^{1}(f), z^{\prime}=$ $j_{y}^{1}(g)$ et que $a\left(z^{\prime}\right)=b(z)$, on peut définir le composé $z^{\prime} \cdot z \in \pi^{1}(M)$ par

$$
z^{\prime} \cdot z=j_{x}^{1}(g \circ f)
$$

et l'inverse $z^{-1} \in \pi^{1}(M)$ par

$$
z^{-1}=j_{f(x)}^{1}\left(f^{-1}\right)
$$

Il est clair que $\pi^{1}(M)$, muni de cette structure du "composé", satisfait aux propriétés suivantes;

1) les applications $a: \pi^{1}(M) \rightarrow M, b: \pi^{1}(M) \rightarrow M$ sont des submersions,

2) l'application $\pi^{1}(M) \ni z \mapsto z^{-1} \in \pi^{1}(M)$ est différentiable,

3) l'application $\pi^{1}(M) \times_{M} \pi^{1}(M) \ni\left(z^{\prime}, z\right) \mapsto z^{\prime} \cdot z \in \pi^{1}(M)$ est différentiable, où $\pi^{1}(M) \times_{M} \pi^{1}(M)$ désigne le fibré produit, i.e,.

$$
\pi^{1}(M) \times_{M} \pi^{1}(M)=\left\{\left(z^{\prime}, z\right) \in \pi^{1}(M) \times \pi^{1}(M) \mid a\left(z^{\prime}\right)=b(z)\right\} .
$$

Soit $\alpha: M \rightarrow N$ une fibration. Considérons un pseudo-groupe $\Gamma$ sur $M$ vérifiant les conditions

$\mathrm{A}_{1}$ ) pour tout $f \in \Gamma$, on a $\alpha \cdot f(x)=\alpha(x)$ quel que soit $x \in U_{f}$,

$\mathrm{A}_{2}$ ) pour tous $x, x^{\prime} \in M$ avec $\alpha(x)=\alpha\left(x^{\prime}\right)$, il existe un élément $f \in \Gamma$ tel que $f(x)=x^{\prime}$.

Posons

$$
\pi^{1}(\Gamma)=\left\{j_{x}^{1}(f) \in \pi^{1}(M) \mid f \in \Gamma, x \in U_{f}\right\}
$$

Comme $\Gamma$ est un pseudo-groupe, on a $z^{-1} \in \pi^{1}(\Gamma)$ et $z^{\prime} \cdot z \in \pi^{1}(\Gamma)$ pour tous $z^{\prime}, z \in \pi^{1}(\Gamma)$ avec $a\left(z^{\prime}\right)=b(z)$.

Definition 1-4. Nous dirons que $\Gamma$ est un pseudo-groupe continu d'ordre 1 sur $M$, si et seulement si les conditions suivantes sont vérifiées;

I) il existe sur $\pi^{1}(\Gamma)$ une structure de sous-variété différentiable de $\pi^{1}(M)$ telle que

1) $a: \pi^{1}(\Gamma) \rightarrow M, b: \pi^{1}(\Gamma) \rightarrow M$ sont des submersions,

2) $\tilde{M}=(a \times b)\left(\pi^{1}(\Gamma)\right)$ est une sous-variété différentiable de $M \times M$ et $a \times b: \pi^{1}(\Gamma) \rightarrow \tilde{M}$ est une submersion, 
3) l'application $\pi^{1}(\Gamma) \ni z \mapsto z^{-1} \in \pi^{1}(\Gamma)$ est différentiable,

4) l'application $\pi^{1}(\Gamma) \times_{M} \pi^{1}(\Gamma) \ni\left(z^{\prime}, z\right) \mapsto z^{\prime} \cdot z \in \pi^{1}(\Gamma)$ est différentiable, où $\pi^{\prime}(\Gamma) \times_{M} \pi^{1}(\Gamma)=\left\{\left(z^{\prime}, z\right) \mid a\left(z^{\prime}\right)=b(z)\right\}$,

II. si $f \in G P(M)$ vérifie $j_{x}^{1}(f) \in \pi^{1}(\Gamma)$ pour tout $x \in U_{f}$, on a $f \in \Gamma$.

En d'autres mots, ces conditions signifient que $\pi^{1}(\Gamma)$ forme l'équation différentielle d'ordre 1 (voir [10], [11]).

Supposons qu'il y ait une section différentiable $I: N \rightarrow M$ par rapport à $\alpha$, et posons

$$
\begin{aligned}
\mathscr{V} & \left.=I^{*} T(M) \text { (i.e., } \mathscr{V}_{\xi}=T_{I(\xi)}(M), \xi \in N\right), \\
G(\Gamma) & =\left\{(\xi, z) \in N \times \pi^{1}(\Gamma) \mid a(z)=b(z)=I(\xi)\right\}, \\
B(\Gamma) & =\left\{(\xi, z) \in N \times \pi^{1}(\Gamma) \mid a(z)=I(\xi)\right\}, \\
\rho: B(\Gamma) & \rightarrow M, \quad \rho(\xi, z)=b(z), \quad(\xi, z) \in B(\Gamma) .
\end{aligned}
$$

LEMME 1-1. $G(\Gamma)$ est un groupe de Lie fibré sur $N$.

En effet, $G(\Gamma)$ est considéré comme le fibré induit de

$$
a \times b: \pi^{1}(\Gamma) \rightarrow \tilde{M} \operatorname{par} \tilde{I}: N \rightarrow \tilde{M}, \quad \tilde{I}(\xi)=(I(\xi), I(\xi)), \quad \xi \in N .
$$

Le composé et l'inverse de $\pi^{1}(\Gamma)$ déterminent sur chaque fibre $G(\Gamma)_{\xi}=$ $G(\Gamma) \cap\{\xi\} \times \pi^{1}(\Gamma)(\xi \in N)$ la structure de groupe. Il est clair que l'application $G(\Gamma) \ni(\xi, z) \mapsto(\xi, z)^{-1} \equiv\left(\xi, z^{-1}\right) \in G(\Gamma)$ est différentiable. $G(\Gamma)$ $\times{ }_{N} G(\Gamma)$, le fibré produit de $G(\Gamma) \rightarrow N,(\xi, z) \mapsto \xi$, est considéré comme une sous-variété différentiable de $N \times\left(\pi^{1}(\Gamma) \times_{M} \pi^{1}(\Gamma)\right)$, et l'application $G(\Gamma)$ $\times_{N} G(\Gamma) \ni\left(w^{\prime}, w\right) \mapsto w^{\prime} \cdot w \in G(\Gamma)$ est la restriction de

$$
N \times\left(\pi^{1}(\Gamma) \times_{M} \pi^{1}(\Gamma)\right) \ni\left(\xi,\left(z^{\prime}, z\right)\right) \mapsto\left(\xi, z^{\prime} \cdot z\right) \in N \times \pi^{1}(\Gamma)
$$

à la sous-variété $G(\Gamma) \times{ }_{N} G(\Gamma)$. Ceci signifie que $G(\Gamma)$ est un groupe de Lie fibré sur $N$.

Ensuite, définissons l'application $\lambda: B(\Gamma) \rightarrow F(M, \alpha, N, \mathscr{V})$ par la formule

$$
\lambda(\xi, z)=\left(f_{*}\right)_{I(\xi)}: \mathscr{V}_{\xi} \rightarrow T_{x}(M) \quad(x=f(I(\xi)))
$$

pour tout $(\xi, z) \in B(\Gamma)$ avec $z=j_{I(\xi)}^{1}(f), f \in \Gamma$, et posons $\lambda^{\prime}=\left.\lambda\right|_{G_{(\Gamma)}}$; par ces applications, $B(\Gamma)$ est considéré comme une sous-variété différentiable de $F(M, \alpha, N, \mathscr{V})$ et $G(\Gamma)$ un sous-groupe de Lie fibré de $G L(\mathscr{V})$. Il est immédiat de constater que $B(\Gamma)_{\xi}=B(\Gamma) \cap\{\xi\} \times \pi^{1}(\Gamma)$ est un fibré principal de base $M_{\xi}=\alpha^{-1}(\xi)$ et de groupe structural $G(\Gamma)_{\xi}$. En vertu de 
$\mathrm{A}_{2}$ ) et de la condition I)-1), il se trouve que $\rho: B(\Gamma) \rightarrow M$ est une submersion.

Nous exprimons ce résultat sous la forme suivante.

THEOREME 1-1. Soit $\Gamma$ un pseudo-groupe continu d'ordre 1 opérant sur $M$ et soit $\alpha: M \rightarrow N$ une fibration. Supposons que $\Gamma$ satisfasse aux conditions $\mathrm{A}_{1}$ ) et $\mathrm{A}_{2}$ ) et qu'il $y$ ait une section différentiable $I$ de $\alpha: M$ $\rightarrow$ N. Posons

$$
\begin{gathered}
\mathscr{V}=I^{*} T(M), \\
B(\Gamma)=\left\{(\xi, z) \in N \times \pi^{1}(\Gamma) \mid a(z)=I(\xi)\right\}, \\
G(\Gamma)=\{(\xi, z) \in B(\Gamma) \mid b(z)=I(\xi)\}, \\
\rho: B(\Gamma) \rightarrow M, \quad \rho(\xi, z)=b(z), \quad(\xi, z) \in B(\Gamma) .
\end{gathered}
$$

Alors $B(\Gamma)$ est considéré naturellement comme une $G \cdot G$-structure de groupe structural $G(\Gamma)$ et de base $(M, \alpha, N, \mathscr{V})$.

Prenons un élément $\phi \in \Gamma$, et définissons l'application local $p(\phi) \in$ $G P(B(\Gamma))$ par

$$
p(\phi)\left(\xi, j_{I(\xi)}^{1}(f)\right)=\left(\xi, j_{I(\xi)}^{1}(\phi \cdot f)\right)
$$

pour tout $\left(\xi, j_{I(\xi)}^{1}(f)\right) \in B(\Gamma)$ tel que $f(I(\xi)) \in U_{\phi}$; on a, de cela, facilement $\rho \cdot p(\phi)=\phi \cdot \rho$. Soit $\theta$ la forme extérieure canonique sur $B(\Gamma)$ (cf. Définition 1-3). Calculons $p(\phi) * \theta$;

$$
\begin{aligned}
\left(p(\phi)^{*} \theta\right)_{\left(\xi, j_{x}^{1}(f)\right)}(X) & =\theta_{\left(\xi, j_{x}^{1}(\phi \cdot f)\right)}\left(p(\phi)_{*}(X)\right) \\
& =\left(\phi_{*} \circ f_{*}\right)^{-1}\left(\rho_{*} \circ p(\phi)_{*} X\right) \\
& =f_{*}^{-1}\left(\rho_{*} X\right) \\
& =\theta_{\left(\xi, j_{x}^{1}(f)\right)}(X),
\end{aligned}
$$

où $x=I(\xi)$ et $X$ désigne un vecteur tangent à $B(\Gamma)$ en $\left(\xi, j_{x}^{1}(f)\right) \in B(\Gamma)$. Donc on obtient, pour tout $\phi \in \Gamma$,

$$
p(\phi) * \theta=\theta .
$$

En tenant compte de cette formule, nous allons définir les isomorphismes des $G \cdot G$-structures.

Soient $(M, \alpha, N, \mathscr{V})$ et $\left(M^{\prime}, \alpha^{\prime}, N^{\prime}, \mathscr{V}^{\prime}\right)$ deux systèmes des bases tels que $\operatorname{dim} M=\operatorname{dim} M^{\prime}, \operatorname{dim} N=\operatorname{dim} N^{\prime}$.

DeFinition 1-5. Un difféomorphisme (local) $\phi$ de $M$ dans $M^{\prime}$ sera 
dit un isomorphisme (local) de $F(M, \alpha, N, \mathscr{V})$ dans $F^{\prime}\left(M^{\prime}, \alpha^{\prime}, N^{\prime}, \mathscr{V}^{\prime}\right)$, s'il existe un difféomorphisme (local) $f$ de $N$ dans $N^{\prime}$ et un isomorphisme local) de fibrés vectoriels $\psi$ de $\mathscr{V}$ dans $\mathscr{V}^{\prime}$ tels que

$$
\alpha^{\prime} \circ \phi=f \circ \alpha, \quad q^{\prime} \circ \psi=f \circ q,
$$

où $q$ (resp. $q^{\prime}$ ) désigne l'application canonique de $\mathscr{V}$ sur $N$ (resp. de $\mathscr{V}^{\prime}$ sur $\left.N^{\prime}\right)$.

Il vaudrait mieux de représenter l'isomorphisme $\phi$ par $\phi=\phi(f, \psi)$. Etant donné un isomorphisme (local) $\phi=\phi(f, \psi)$, nous définissons une application (locale) $\tilde{\phi}$ de $F(M, \alpha, N, \mathscr{V})$ dans $F^{\prime}\left(M^{\prime}, \alpha^{\prime}, N^{\prime}, \mathscr{V}^{\prime}\right)$ par

$$
\tilde{\phi}(z)=\phi_{*} \circ z \circ \psi^{-1}: \mathscr{V}_{\xi} \rightarrow T_{\phi(x)}(M)
$$

$(z \in F, x=\rho(z), \xi=\alpha(x))$. Il est facile de démontrer que $\tilde{\phi}$ est un difféomorphisme (local) de $F$ dans $F^{\prime}$ tel que

$$
\rho^{\prime} \circ \tilde{\phi}=\phi \circ \rho \text {. }
$$

Si $\psi$ est un isomorphisme (local) de $\mathscr{V}$ dans $\mathscr{V}^{\prime}$ avec $q^{\prime} \circ \psi=f \circ q$, nous désignons par Ad $(\psi)$ l'isomorphisme de groupes de Lie fibrés de $G L(\mathscr{V})$ dans $G L\left(\mathscr{V}^{\prime}\right)$ tel que, pour tout $g \in G L(\mathscr{V})$ avec $\pi(g) \in U_{f}$,

$$
\operatorname{Ad}(\psi)(g)=\psi \circ g \circ \psi^{-1}
$$

et que

$$
\pi^{\prime} \circ \operatorname{Ad}(\psi)=f \circ \pi .
$$

Pour un isomorphisme $\phi=\phi(f, \psi)$, on a donc

$$
\tilde{\phi}(z \cdot g)=\phi(z) \operatorname{Ad}(\psi)(g), \quad\left((z, g) \in F \times_{N} G L(\mathscr{V})\right) .
$$

Soit $B=B(M, \alpha, N, \mathscr{V})$ une $G \cdot G$-structure de groupe structural $G$ et soit $B^{\prime}=B^{\prime}\left(M^{\prime}, \alpha^{\prime}, N^{\prime}, \mathscr{V}^{\prime}\right)$ une $G \cdot G$-structure de groupe structural $G^{\prime}$.

Definition 1-6. Nous dirons qu'un isomorphisme (local) $\phi=\phi(f, \psi)$ est un isomorphisme (local) de $B$ dans $B^{\prime}$, si et seulement si l'application induite $\tilde{\phi}$ vérifie $\tilde{\phi}\left(\left.B\right|_{U_{\phi}}\right)=\left.B^{\prime}\right|_{V_{\phi}}$.

Definition 1-7. Si, pour tous $x \in M, y \in M^{\prime}$, il existe un isomorphisme local $\phi=\phi(f, \psi)$ de $B$ dans $B^{\prime}$ tel que $\phi(x)=y, B$ et $B^{\prime}$ seront dits localement isomorphes.

Proposition 1-4. Soit $\phi=\phi(f, \psi)$ un isomorphisme (local) de $B$ 
dans $B^{\prime}$ et soient $\theta, \theta^{\prime}$ les formes extérieures canoniques sur $B$ et sur $B^{\prime}$ respectivement. Alors l'application induite $\tilde{\phi}$ vérifie

$$
\tilde{\phi}^{*} \theta^{\prime}=\psi \circ \theta
$$

DeFinition 1-8. Un isomorphisme (local) $\phi=\phi(f, \psi)$ de $B$ dans $B$ sera dit un automorphisme (local) invariant de $B$. Un automorphisme (local) invariant $\phi=\phi(f, \psi)$ de $B$ vérifiant $\psi=\mathrm{id}$ sera dit un $N$-automorphisme (local) de $B$ (voir (1-1) et Proposition 1-4).

Désignons par $\Gamma^{*}(B)$ l'ensemble de tout automorphisme local invariant de $B$ et par $\Gamma(B)$ l'ensemble de tout $N$-automorphisme de $B$.

Etant donnés, en général, deux pseudo-groupes $\Gamma$ et $\Gamma^{\prime}$ sur $M$ et sur $M^{\prime}$ respectivement. Un difféomorphisme local $\phi$ de $M$ dans $M^{\prime}$ est dit un isomorphisme local de $\Gamma$ dans $\Gamma^{\prime}$, si et seulement si la condition suivante est vérifiée;

$$
\operatorname{ad}(\phi)\left(\left.\Gamma\right|_{U_{\phi}}\right)=\left.\Gamma\right|_{V_{\phi}},
$$

où $\left.\Gamma\right|_{U_{\phi}}=\left\{f \in \Gamma \mid U_{f}, V_{f} \subset U_{\phi}\right\}$ et $\operatorname{ad}(\phi)(f)=\phi \circ f \circ \phi^{-1},\left.f \in \Gamma\right|_{U_{\phi}}$.

On dit, de plus, que $\Gamma$ et $\Gamma^{\prime}$ sont localement isomorphes, si, pour tous $x \in M, y \in M^{\prime}$, il existe un isomorphisme local de $\Gamma$ dans $\Gamma^{\prime}$ tel que $\phi(x)=y . \quad N(\Gamma)$, l'ensemble de tout isomorphisme local de $\Gamma$ dans $\Gamma$, est dit le normalisateur de $\Gamma$.

Proposition 1-5. Soit $B(M, \alpha, N, \mathscr{V})$ une $G \cdot G$-structure. Alors le normalisateur $N(\Gamma(B))$ du pseudo-groupe des $N$-automorphismes locals de $B$ contient le pseudo-groupe $\Gamma^{*}(B)$, i.e.,

$$
\Gamma^{*}(B) \subset N(\Gamma(B))
$$

En effet, soit $\phi=\phi(f, \psi) \in \Gamma^{*}(B)$; il faut établir $\operatorname{ad}(\phi)\left(\left.\Gamma(B)\right|_{U_{\phi}}\right)=$ $\left.\Gamma(B)\right|_{V_{\phi}}$, mais, en vertu de la relation $\widetilde{\phi \circ g \circ \phi^{-1}}=\tilde{\phi} \circ \tilde{g} \circ \tilde{\phi}^{-1}, P=\phi \circ g \circ \phi^{-1}$ et $P^{-1}$ transportent $B$ dans $B$ pour tout $\left.g \in \Gamma(B)\right|_{U_{\phi}}$ Soit $\theta$ la forme extérieure canonique sur $B$. D'après la Proposition $1-4$, on a

$$
\tilde{P}^{*} \theta=\tilde{\phi}^{-1 *} \circ \tilde{g}^{*} \circ \tilde{\phi}^{*} \theta=\psi\left(\tilde{\phi}^{-1 *} \circ \tilde{g}^{*} \theta\right)=\psi\left(\tilde{\phi}^{-1 *} \theta\right),
$$

par suite, $\tilde{P}^{*} \theta=\theta$, puisque $\tilde{\phi}^{-1 *} \theta=\psi^{-1} \circ \theta$. Donc l'isomorphisme de fibrés vectoriels associé à $P$ est trivial.

De la même manière, nous pouvons démontrer le théorème suivant.

THEOREME 1-2. Soient $B(M, \alpha, N, \mathscr{V})$ et $B^{\prime}\left(M^{\prime}, \alpha^{\prime}, N^{\prime}, \mathscr{V}^{\prime}\right)$ des $G \cdot G-$ 
structures. Si $\phi=\phi(f, \psi)$ est un isomorphisme local de $B$ dans $B^{\prime}, \phi$ est un isomorphisme local de $\Gamma(B)$ dans $\Gamma\left(B^{\prime}\right)$. En conséquence, si $B$ et $B^{\prime}$ sont localement isomorphes, $\Gamma(B)$ et $\Gamma\left(B^{\prime}\right)$ sont aussi localement isomorphes.

D'après ce théorème, nous pouvons partager le problème d'équivalence des pseudo-groupes en deux.

En premier lieu, si un pseudo-groupe $\Gamma$ est donné sur $M$, il faut trouver une $G \cdot G$-structure telle que $\Gamma$ coïncide avec le pseudo-groupe des $N$-automorphismes. Le Théorème 1-1 a quelque relation à ce problème.

En deuxième lieu, nous sommes ramenés à la recherche des invariants $\operatorname{des} G \cdot G$-structures vis-à-vis des automorphismes invariants.

Dans les paragraphes suivants, nous étudierons ce deuxième problème. Dans notre cas, il se trouve que la forme extérieure canonique $\theta$ sur $B$ est inutile pour le problème. C'est parce que d'une part il faut trouver à la fois trois applications et que d'autre part, un automorphisme invariant ne laisse pas toujours $\theta$ invariant (cf. Proposition 1-4).

Pour suivir la méthode de Cartan ([2], [4]), il est nécessaire de construire un autre espace associé à la $G \cdot G$-structure donnée de manière que, dans cet espace, on puisse définir une forme extérieure invariante par les automorphismes invariants.

\section{§2. $G_{0}^{*}$-structures associées aux $G \cdot G$-structures}

Dans ce paragraphe, pour étudier le problème d'équivalence, nous allons construire une structure géométrique associée à une $G \cdot G$-structure et établir la relation entre les deux structures. Nous commençons par donner quelques notions qui sont utilisées dans la suite.

Soit $V$ un espace vectoriel réel de dimension finie et soit $W$ un sousespace vectoriel de $V$.

DEFINITION 2-1. $G$ étant un sous-groupe de Lie de $G L(V)$, le système $(V, W, G)$ sera dit compatible, si chaque élément $g$ de $G$ vérifie $\pi \circ g=\pi$, où $\pi$ désigne l'application canonique $V \rightarrow V / W$.

DEFINITION 2-2. (Ss étant une sous-algèbre de Lie de gl( $V)$, le système $(V, W$, (S) sera dit compatible, si chaque élément $X$ de \&s vérifie $\pi \circ X=0$. En conséquence, on a \& $\subset \operatorname{Hom}(V, W)$. 
Soit $(V, W, G)$ un système compatible et soit đs' l'algébre de Lie de $G$, et posons

$$
\begin{aligned}
& G^{*}=\left\{g \in G L(V) \mid g G g^{-1}=G, g(W)=W\right\}, \\
& \text { (্)* }=\{X \in g h(V) \mid[X, \mathbb{S}] \subset \mathbb{S}, X(W) \subset W\} .
\end{aligned}
$$

Il est clair que $G^{*}$ est un groupe de Lie et que (5* est son algébre de Lie. Considérons, de plus, l'espace vectoriel $\mathbb{S}^{*} \oplus V$, la somme directe, et définissons un sous-groupe de Lie $G^{*}$ de $G L\left(\left(^{*} \oplus V\right)\right.$ par la condition suivante :

$h \in G L(\varangle 3 * \oplus V)$ appartient à $G^{\#}$ si et seulement si

i) $h(A)=A \quad$ pour tout $A \in$ (S),

ii) $h(W)=W$,

iii) $V$-composant de $\left.h\right|_{V}$ est à $G$,

sont vérifiés. Passons cette condition à son algéble (s) $\subset$ gl((ङ)* $\oplus V)$. Il en résulte que $\left.X \in \mathfrak{g r}(\circlearrowleft)^{*} \oplus V\right)$ appartient à (ك)\# si et seulement si

i) $X\left(\mathbb{S}^{*}\right)=\{0\}$,

ii) $\quad X(W) \subset W$,

iii) $\quad V$-composant de $\left.X\right|_{V}$ est à đ্s,

sont vérifiés. Il est clair que $\mathbb{S}^{\sharp}$ est isomorphe à l'espace vectoriel $\operatorname{Hom}(V / W$, (s) $) \oplus$ (s), i.e.,

$$
\text { (3) } \cong \operatorname{Hom}(V / W, \text { (s) }) \oplus \text { (s) . }
$$

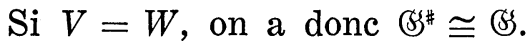

Ensuite, soit $\mathscr{V}$ un fibré vectoriel réel sur une variété différentiable $N$ et soit $\tilde{n}$. la dimension des fibres de $\mathscr{V}$. Désignons par $\Pi(\mathscr{V})$ l'ensemble de toutes les applications linéaires isomorphes de $\boldsymbol{R}^{\tilde{n}}$ sur chaque fibre de $\mathscr{V}$ et $a: \Pi(\mathscr{V}) \rightarrow N$ la projection canonique. Alors, on peut retrouver facilement que $\Pi(\mathscr{V})$ admet une structure canonique d'espace fibré principal différentiable, de base $N$ et de groupe structural $G L(\tilde{n}, \boldsymbol{R})$.

Soient $\alpha: M \rightarrow N$ une fibration, $\mathscr{V}$ un fibré vectoriel réel de base $N$ tel que pour tout $\xi \in N$,

$$
\operatorname{dim} M=\operatorname{dim} \mathscr{V}_{\xi}=m+n
$$

soit vérifié; et notons les par $(M, \alpha, N, \mathscr{V})$. Etant donnés un sous-groupe de Lie fibré $G$ de $G L(\mathscr{V})$ et un système compatible $\left(V, W, G_{0}\right)$ vérifiant les relations $\operatorname{dim} M=\operatorname{dim} V$ et $\operatorname{dim} M-\operatorname{dim} N=\operatorname{dim} W$, nous posons la 
Definition 2-3. Nous dirons qu'une $G \cdot G$-structure $B$, de base $(M, \alpha, N, \mathscr{V})$ et de groupe structural $G$, est de type $\left(V, W, G_{0}\right)$, si et seulement si les conditions suivantes sont vérifiées;

(1) pour tous $z, z^{\prime} \in B$ avec $\alpha \circ \rho(z)=\alpha \circ \rho\left(z^{\prime}\right), \alpha_{*} \circ z=\alpha_{*} \circ z^{\prime}$ est vérifié, et $\alpha_{*} \circ z=\gamma_{\xi}: \mathscr{V}_{\xi} \rightarrow T_{\xi}(N)(z \in B, \xi=\alpha \circ \rho(z))$ détermine un homomorphisme surjectif de fibrés vectoriels $\gamma: \mathscr{V} \rightarrow T N$,

(2) $\Pi(\mathscr{V}, G)=\left\{u \in \Pi(\mathscr{V}) \mid u \circ G_{0} \circ u^{-1}=G_{a(u)}, \gamma \circ u(W)=\{0\}\right\}$ est un sousfibré principal différentiable de $\Pi(\mathscr{V})$, de groupe structural $G_{0}^{*}$.

D'après cette définition, on a aisément le

LEMME 2-1. a) $\gamma_{\xi} \circ g=\gamma_{\xi}$, pour tout $g \in G$ avec $\pi(g)=\xi$, b) $u(W)=\left.\operatorname{Ker} \gamma\right|_{a(u)}$, pour tout $u \in \Pi(\mathscr{V}, G)$.

Soit $B(M, \alpha, N, \mathscr{V})$ une $G \cdot G$-structure de groupe structural $G$. Supposons que $B$ soit de type $\left(V, W, G_{0}\right)$ et posons

$$
M(G)=\{(u, x) \in \Pi(\mathscr{V}, G) \times M \mid a(u)=\alpha(x)\} .
$$

Comme $M(G)$ est le fibré produit de $a: \Pi(\mathscr{V}, G) \rightarrow N$ et de $\alpha: M \rightarrow N$, c'est une sous-variété différentiable de $\Pi(\mathscr{V}, G) \times M$. Nous désignons par $\lambda$ (resp. $\mu$ ) l'application (submersion) de $M(G)$ sur $\Pi(\mathscr{V}, G)($ resp. $M$ );

$$
\begin{gathered}
\lambda: M(G) \rightarrow \Pi(\mathscr{V}, G): \lambda(u, x)=u \\
\text { (resp. } \mu: M(G) \rightarrow M: \mu(u, x)=x),
\end{gathered}
$$

pour tout $(u, x) \in M(G)$. Par suite, on a

$$
a \circ \lambda=\alpha \circ \mu \text {. }
$$

Comme $G_{0}^{*}$ est le groupe structural du fibré principal $\Pi(\mathscr{V}, G), G_{0}^{*}$ peut opérer non seulement sur $\Pi(\mathscr{V}, G)$ mais aussi sur $M(G)$. Par ces opérations de $G_{0}^{*}, A \in \mathbb{S}_{0}^{*}$ entraîne les champs de vecteurs sur $\Pi(\mathscr{V}, G)$ et sur $M(G)$; notons les par la même lettre $A^{*}$. Alors, on a

$$
\lambda_{*} A_{s}^{*}=A_{\lambda(s)}^{*}, \quad \mu_{*} A_{s}^{*}=0, \quad a_{*} A_{u}^{*}=0,
$$

pour tous $s \in M(G), u \in \Pi(\mathscr{V}, G)$.

Proposition 2-1. Si $\pi(\mathscr{V}, G)$ est trivial, i.e., $N \times G_{0}^{*} \cong \Pi(\mathscr{V}, G)$, il existe un difféomorphisme $\phi$ de $M \times G_{0}^{*}$ sur $M(G)$ tel que $\mu \circ \phi(x, g)=x$, $\lambda \circ \phi(x, g)=\nu(\alpha(x), g)$ pour tout $(x, g) \in M \times G_{0}^{*}$, où $\nu$ désigne l'isomorphisme $N \times G_{0}^{*} \rightarrow \Pi(\mathscr{V}, G)$. 
En effet, on n'a qu'à définir l'application $\phi: M \times G_{0}^{*} \rightarrow M(G)$ par $\phi(x, g)=(\nu(\alpha(x), g), x)$ pour $(x, g) \in M \times G_{0}^{*}$.

Désignons par $P(B)$ l'ensemble de toute application linéaire isomorphe $X$ de $\mathbb{S}_{0}^{*} \oplus V$ sur $T_{s}(M(G)$ ) (pour tout $s \in M(G)$ ) possédant les propriétés;

i) $X(A)=A_{s}^{*} \quad$ pour tout $A \in \mathbb{B}_{0}^{*}$,

ii) $\lambda_{*}^{0} X(w)=0 \quad$ pour tout $w \in W$,

iii) $\Phi(X)=\mu_{*} \circ X \circ(\lambda(s))^{-1}: \quad \mathscr{V}_{\xi} \rightarrow T_{x}(M)(x=\mu(s), \xi=\alpha(x))$ est un isomorphisme et appartient à $B$.

Ce que nous voulons faire dans la suite, c'est de démontrer que $P(B)$ est un sous-fibré principal différentiable de l'espace des repères de $M(G)$, de groupe structural $G_{0}^{\#}$. Nous allons commencer par construire des sections différentiables locales de $\varpi: F(M(G)) \rightarrow M(G)$ à valeurs dans $P(B)$.

Dans la considération suivante, nous reprendrons chaque voisinage plus petit s'il en est besoin, sans l'exprimer distinctement. Soient $\left(x^{1}, \cdots, x^{n}, y^{1}, \cdots, y^{m}\right)$ un système de coordonnées locales de $U \subset M$ et $\left(\bar{x}^{1}, \cdots, \bar{x}^{n}\right)$ celui de $U^{\prime}=\alpha(U) \subset N$ tels que $\bar{x}^{i} \circ \alpha=x^{i}(i=1,2, \cdots, n)$. Supposons que $\left.\Pi(\mathscr{V}, G)\right|_{U^{\prime}}$ soit trivial, c'est-à-dire qu'il y ait un isomorphisme $\psi: U^{\prime} \times\left. G_{0}^{*} \rightarrow \Pi(\mathscr{V}, G)\right|_{U^{\prime}}$. D'après la Proposition 2-1, il existe un difféomorphisme $\phi: U \times\left. G_{0}^{*} \rightarrow M(G)\right|_{U}$. Soient $\sigma_{1}, \cdots, \sigma_{n+m}$ des sections différentiables linéairement indépendantes de $\left.\mathscr{V}\right|_{U}$, telles que

$$
\begin{array}{ll}
\gamma_{\xi}\left(\sigma_{i}(\xi)\right)=0 & (i=1,2, \cdots, m), \\
\gamma_{\xi}\left(\sigma_{m+j}(\xi)\right)=\left(\frac{\partial}{\partial \bar{x}^{j}}\right)_{\xi} & (j=1,2, \cdots, n),
\end{array}
$$

pour tout $\xi \in U^{\prime}$ (cf. Définition 2-3). Prenons une section différentiable $\tau: U \rightarrow B$ représentée par les formules,

$$
\begin{aligned}
\tau(x)\left(\sigma_{i}(\xi)\right) & =\sum_{k=1}^{m} A_{i}^{k}(x)\left(\frac{\partial}{\partial y^{k}}\right)_{x} & (i=1,2, \cdots, m), \\
\tau(x)\left(\sigma_{m+j}(\xi)\right) & =\left(\frac{\partial}{\partial x^{j}}\right)_{x}+\sum_{k=1}^{m} A_{m+j}^{k}(x)\left(\frac{\partial}{\partial y^{k}}\right)_{x} & (j=1,2, \cdots, n),
\end{aligned}
$$

pour tout $x \in U, \alpha(x)=\xi$, où $A_{i}^{k}$ sont des fonctions différentiables. Soit $\left\{\boldsymbol{a}_{1}, \cdots, \boldsymbol{a}_{m+n}\right\}$ un système de base de l'espace vectoriel $V$ tel que $\left\{\boldsymbol{a}_{1}, \cdots\right.$, $\left.\boldsymbol{a}_{m}\right\}$ soit un système de base de $W$. Définissons une section différentiable $\tilde{\tau}:\left.M(G)\right|_{U} \rightarrow F(M(G))$ par 
$1^{\circ} \quad \tilde{\tau}(s)(A)=A_{s}^{*} \quad A \in \mathbb{S}_{0}^{*}$,

$2^{\circ} \quad \tilde{\tau}(s)\left(\boldsymbol{a}_{i}\right)=\left(\phi_{e}\right)_{*} \circ \tau(\mu(s)) \circ \lambda(s)\left(\boldsymbol{a}_{i}\right) \quad(i=1,2, \cdots, n+m)$

pour tout $\left.s \in M(G)\right|_{U}$, où $\phi_{e}$ désigne l'application induite de $\phi ; \phi_{e}(x)=$ $\phi(x, e), x \in U, e$ l'élément neutre de $G_{0}^{*}$.

LEMME 2-2. $\quad \tilde{\tau}:\left.M(G)\right|_{U} \rightarrow F(M(G))$ est une section différentiable vérifiant $\tilde{\tau}\left(\left.M(G)\right|_{U}\right) \subset P(B)$.

En effet, d'après la Proposition 2-1, on a $\lambda \circ \phi_{e}(x)=\psi_{e} \circ \alpha(x)(x \in U)$ en posant $\psi_{e}(\xi)=\psi(\xi, e), \xi \in V$. De la définition de $\Pi(\mathscr{V}, G)$, on peut représenter $\lambda(s)\left(\boldsymbol{a}_{i}\right)(i=1,2, \cdots, m)$ par

$$
\lambda(s)\left(\boldsymbol{a}_{i}\right)=\sum_{j=1}^{m} B_{i}^{j}(s) \sigma_{j}(\xi) \quad(\xi=\alpha \circ \mu(s)) .
$$

Donc on a, à l'aide de la reration $\lambda \circ \phi_{e}=\psi_{e} \circ \alpha$ et de $2^{\circ}$,

$$
\begin{aligned}
\lambda_{*} \circ \tilde{\tau}(s)\left(\boldsymbol{a}_{i}\right) & =\left(\psi_{e}\right)_{*} \circ \alpha_{*} \circ \tau(\mu(s))\left(\lambda(s)\left(\boldsymbol{a}_{i}\right)\right) \\
& =\sum_{\substack{j=1 \\
k=1}}^{m} B_{i}^{j}(s) A_{j}^{k}(\mu(s))\left(\psi_{e}\right)_{*} \circ \alpha_{*}\left(\frac{\partial}{\partial y^{k}}\right)_{\mu(s)} \\
& =0 \quad(i=1,2, \cdots, m) .
\end{aligned}
$$

Ensuite, il faut trouver $\Phi(\tilde{\tau}(s))=\tau(\mu(s)) \in B$ pour tout $\left.S \in M(G)\right|_{U}$. Mais il résulte de la relation $\mu \circ \phi_{e}=$ id sur $U$.

Déterminons les sous-espaces vectoriels de $T_{s}(M(G))$;

$$
\begin{aligned}
& T_{s}^{0}(\mu)=\left\{v \in T_{s}(M(G)) \mid \mu_{*}(v)=0\right\}, \\
& T_{s}^{0}(\lambda)=\left\{v \in T_{s}(M(G)) \mid \lambda_{*}(v)=0\right\} .
\end{aligned}
$$

LEMME 2-3. Tout $X \in P(B)$ définit canoniquement les isomorphismes linéaires

$$
\begin{aligned}
& X: \mathbb{S}_{0}^{*} \rightarrow T_{s}^{0}(\mu), \\
& X: W \rightarrow T_{s}^{0}(\lambda) .
\end{aligned}
$$

Ce lemme résulte de la définition de $P(B)$.

LEMME 2-4. $G_{0}^{\#}$ est le groupe structural de $P(B)$.

Supposons que $g$ soit un élément de $G L\left(\mho^{*} \oplus V\right)$ tel que $X \circ g \in P(B)$ pour tout $X \in P(B)$. D'abord, pour tout $A \in \mathbb{S}_{0}^{*}$, on a $(X g)(A)=A_{s}^{*}$ et $X(g(A))=(g(A))_{s}^{*}(s=\widetilde{\varpi}(X))$, par suite, $g(A)=A$, puisque $(X g)(A)=$ $X(g A)$. Puis, pour tout $w \in W, 0=\lambda_{*} \circ(X g)(w)=\lambda_{*} \circ X(g(w))$. Par conséquent, $X(g(w))$ appartient à $T_{s}^{0}(\lambda)$. D'après le lemme 2-3 ii), il existe 
$\tilde{w} \in W$ tel que $X(\tilde{w})=X(g(w))$. Il en résulte que $g(W)=W$.

Posons $s=\varpi(X), u=\lambda(s), x=\mu(s)$ et $\xi=\alpha(x)$. Comme $\Phi(X)$ et $\Phi(X g)$ sont les isomorphismes de $\mathscr{V}_{\xi}$ sur $T_{x}(M)$, il existe un élément $h_{\xi} \in G_{\xi}$ tel que $\mu_{*} \circ X\left(u^{-1} \circ h_{\xi} \circ u\right)(v)=\mu_{*}(X g)(v)$ pour tout $v \in V$. En posant $h=u^{-1}$ $\circ h_{\xi} \circ u$, on a $h \in G_{0}$. D'après le lemme 2-3 i) et la relation $\mu_{*} \circ(X(g(v)$ $-h(v)))=0$, il existe $A_{v} \in \mathbb{S}_{0}^{*}$ vérifiant $X(g(v)-h(v))=X\left(A_{v}\right)$. Par suite, $g(v)=A_{v}+h(v)$.

Cela signifie que $G_{0}^{\#}$ est le groupe structural de $P(B)$.

Nous exprimerons ce résultat sous la forme suivante:

Proposition 2-2. Soit $B(M, \alpha, N, \mathscr{V})$ une $G \cdot G$-structure de groupe structural $G$ et de type $\left(V, W, G_{0}\right)$. Posons

$$
M(G)=\{(u, x) \in \pi(\mathscr{V}, G) \times M \mid a(u)=\alpha(x)\} .
$$

Alors $M(G)$ admet une G-structure $P(B)$ de groupe structural $G_{0}^{\#}$ vérifiant les conditions suivantes;

i) $X(A)=A_{s}^{*} \quad A \in \mho_{0}^{*}$,

ii) $\lambda_{*} \circ X(W)=0 \quad w \in W$,

iii) $\Phi(X)=\mu_{*} \circ X \circ(\lambda(s))^{-1}: \mathscr{V}_{\xi} \rightarrow T_{x}(M)(x=\mu(s), \xi=\alpha(x))$ est un isomorphisme et appartient à $B$,

pour tout $X \in P(B)$ avec $s=\varpi(X)$.

Voici maintenant nous énonçons le théorème fondamental qui donne la relation entre $B$ et $P(B)$.

THEOREME 2-1. Soient $B(M, \alpha, N, \mathscr{V})$ et $B^{\prime}\left(M^{\prime}, \alpha^{\prime}, N^{\prime}, \mathscr{V}^{\prime}\right)$ des $G \cdot G$ structures de type $\left(V, W, G_{0}\right)$ et de groupes structurals $G$ et $G^{\prime}$, respectivement. Alors tout isomorphisme local $\phi=\phi(f, \psi)$ de $B$ dans $B^{\prime}$ entraîne l'isomorphisme local Ad $(\psi): G \rightarrow G^{\prime}$ et l'isomorphisme $\psi \wedge \phi$ des deux structures $P(B)$ et $P\left(B^{\prime}\right)$, défini par $(\psi \wedge \phi)(u, x)=(\psi \circ u, \phi(x))$ pour $(u, x) \in M(G)$ avec $x \in U_{\phi}$.

Réciproquement, soit $F$ un isomorphisme local des deux structures $P(B)$ et $P\left(B^{\prime}\right)$. Si $G_{0}^{*}$ est connexe, il existe, pour chaque point du domaine de $F$, un isomorphisme local $\phi=\phi(f, \psi)$ de $B$ dans $B^{\prime}$ tel que $F$ coïncide avec $\psi \wedge \phi$ autour de ce point.

Laissons la démonstration de ce théorème pour le moment et énonçons de plus, les théorèmes provenants tout de suite de ce théorème. 
THEOREME 2-2. On va utiliser les notations dans le théorème précédant. Si les deux G-structures $P(B)$ et $P\left(B^{\prime}\right)$ sont localement isomorphes et que $G_{0}^{*}$ soit connexe, alors $B$ et $B^{\prime}$ sont localement isomorphes. Par conséquent, les pseudo-groupes $\Gamma(B)$ et $\Gamma\left(B^{\prime}\right)$ sont aussi localement isomorphes.

Ce théorème résulte du Théorème 1-2 et du Théorème 2-1.

THEOREME 2-3. Soit $B(M, \alpha, N, \mathscr{V})$ une $G \cdot G$-structure de groupe structural $G$. Supposons que $B$ soit de type $\left(V, W, G_{0}\right)$ et que $G_{0}^{*}$ soit connexe. Si le pseudo-groupe des automorphismes de $P(B)$ opère transitivement sur $M(G)$, le normalisateur de $\Gamma(B), N(\Gamma(B))$, est transitif sur $M$.

En effet, soient $x$ et $y$ deux points arbitraires de $M$; il existe deux points $s, t \in M(G)$ tels que $\mu(s)=x, \mu(t)=y$, et un isomorphisme local $F$ des structures $P(B)$ et $P\left(B^{\prime}\right)$ tel que $F(s)=t$. D'après le Théorème 2-1, on peut supposer que $F$ coïncide avec $\psi \wedge \phi$, où $\phi=\phi(f, \psi)$ est un automorphisme invariant de $B$, i.e., $\phi \in \Gamma^{*}(B)$. On a $\phi(x)=y$, puisque $\mu \circ F=\phi \circ \mu$. L'assertion résulte de la relation $\Gamma^{*}(B) \subset N(\Gamma(B))$.

CoRollaIRe 2-1. On désigne par $\Gamma_{\xi}(B)(\xi \in N)$ la restriction $d u$ pseudo-groupe $\Gamma(B)$ à la fibre $M_{\xi}=\alpha^{-1}(\xi)$. Si toutes les conditions dans le Théorème 2-3 sont vérifiées, $\Gamma_{\xi}(B), \xi \in N$, sont localement isomorphes entre eux.

Démonstration du Théorème 2-1.

Soit $\phi=\phi(f, \psi)$ un isomorphisme de $B$ dans $B^{\prime}$. Pour simplifier les notations, supposons que $\phi$ soit défini globalement sur $M$. D'abord, trouvons que l'application $\tilde{\psi}: \Pi(\mathscr{V}) \rightarrow \Pi\left(\mathscr{V}^{\prime}\right)$, définie par $\tilde{\psi}(u)=\psi \circ u$ $(u \in \Pi(\mathscr{V}))$, entraîne l'isomorphisme de fibrés principaux $\tilde{\psi}: \Pi(\mathscr{V}, G) \rightarrow$ $\Pi\left(\mathscr{V}^{\prime}, G^{\prime}\right)$. On a facilement $\operatorname{Ad}(\psi)(G)=G^{\prime}$, puisque $\tilde{\phi}(B)=B^{\prime}$ (cf. 1-2). Par suite, on a, pour tout $u \in \Pi(\mathscr{V}, G)$,

$$
\begin{aligned}
\tilde{\psi}(u) \circ G_{0} \circ \tilde{\psi}(u)^{-1} & =\operatorname{Ad}(\psi)\left(G_{a(u)}\right) \\
& =G_{a^{\prime}(\tilde{\psi}(u))}^{\prime},
\end{aligned}
$$

et on a aussi, pour tout $u \in \Pi(\mathscr{V}, G)$ avec $\xi=a(u), \xi^{\prime}=f(\xi)$,

$$
\gamma_{\xi^{\prime}}^{\prime} \circ \tilde{\psi}(u)(W)=\gamma_{\xi^{\prime}}^{\prime} \circ \psi \circ u \circ(W)
$$




$$
\begin{aligned}
& =\alpha_{*}^{\prime} \circ \tilde{\phi}(z) \circ \psi \circ u(W) \\
& =f_{*} \circ \alpha_{*} \circ z \circ \psi^{-1} \circ \psi \circ u(W) \\
& =f_{*} \circ \gamma_{\xi} \circ u(W)=0,
\end{aligned}
$$

où $z$ est un élément de $B$ tel que $\alpha \circ \rho(z)=\xi$. Ceci démontre $\tilde{\psi}(\Pi(\mathscr{V}, G))$ $=\Pi\left(\mathscr{V}^{\prime}, G^{\prime}\right)$. Donc on peut définir un difféomorphisme $\psi \wedge \phi: M(G) \rightarrow$ $M^{\prime}\left(G^{\prime}\right)$ par la formule $(\psi \wedge \phi)(u, x)=(\tilde{\psi}(u), \phi(x)),(u, x) \in M(G)$. Il est immédiat de constater le

LEMME 2-5. Le difféomorphisme $\psi \wedge \phi$ posséde les propriétés

i) $\lambda^{\prime} \circ(\psi \wedge \phi)=\tilde{\psi} \circ \lambda$

ii) $\mu^{\prime} \circ(\psi \wedge \phi)=\phi \circ \mu$

iii) $R_{g}^{\prime} \circ(\psi \wedge \phi)=(\psi \wedge \phi) \circ R_{g}, g \in G_{0}^{*}$,

oú $R_{g}$ désigne l'opération de $G_{0}^{*}$ sur $M(G)$ et $R_{g}^{\prime}$ celle de $G_{0}^{*}$ sur $M^{\prime}\left(G^{\prime}\right)$.

A l'aide de ce lemme, prouvons que l'application $\psi \wedge \phi: F(M(G)) \rightarrow$ $F\left(M^{\prime}\left(G^{\prime}\right)\right.$ ) vérifie $\overparen{\psi \wedge \phi}(P(B))=P\left(B^{\prime}\right)$. Pour cela, il faut établir pour tout $X \in P(B)$

a) $((\widetilde{\psi \wedge \phi})(X))(A)=A_{(\psi \wedge \phi)(\alpha(X))}^{*} \quad A \in \mathbb{S}_{0}^{*}$,

b) $\lambda_{*}^{\prime} \circ((\widetilde{\psi \wedge \phi})(X))(w)=0 \quad w \in W$,

c) $J(X) \equiv \mu_{*}^{\prime}((\widetilde{\psi \wedge \phi})(X))\left(\lambda^{\prime}\left(\widetilde{\varpi}^{\prime}(\widetilde{\psi \wedge \phi})(X)\right)\right)^{-1} \in B$.

Mais, a) résulte de Lemme 2-5 iii), b) résulte du Lemme 2-5 i). Calculons, à l'aide du Lemme 2-5 ii) et de la définition de $\tilde{\phi}$, le premièr terme de c); on a

$$
\begin{aligned}
& J(X)=\mu_{*}^{\prime}(\psi \wedge \phi)_{*} \circ X \circ\left(\lambda^{\prime} \circ(\widetilde{\psi \wedge \phi}) \circ(\varpi(X))^{-1}\right. \\
& =\phi_{*} \circ \mu_{*} \circ X \circ(\psi \circ(\lambda \circ \widetilde{\sigma}(X)))^{-1} \\
& =\tilde{\phi}\left(\mu_{*} \circ X(\lambda(\varpi(X)))^{-1}\right) \text {, }
\end{aligned}
$$

par suite, $J(X) \in B^{\prime}$, puisque $\tilde{\phi}(B)=B^{\prime}, \mu_{*} \circ X(\lambda(\varpi(X)))^{-1} \in B$. Donc on a $(\widetilde{\psi \wedge \phi})(P(B))=P\left(B^{\prime}\right)$. Ceci démontre que $\phi(f, \psi)$ entraîne l'isomorphisme local $\psi \wedge \phi$ de $P(B)$ dans $P\left(B^{\prime}\right)$.

Nous avons démontré aussi le

LEMME 2-6. Soit $\phi=\phi(f, \psi)$ un isomorphisme (local) de $F(M, \alpha, N$, $\mathscr{V})$ dans $F^{\prime}\left(M^{\prime}, \alpha^{\prime}, N^{\prime}, \mathscr{V}^{\prime}\right)$. Supposons que l'application $\psi \wedge \phi$, définie par la formule $(\psi \wedge \phi)(u, x)=(\psi \circ u, \phi(x)),(u, x) \in M(G)$, soit un difféomorphisme (local) de $M(G)$ dans $M^{\prime}\left(G^{\prime}\right)$ et en outre, que $\psi \wedge \phi$ soit un isomorphisme (local) des deux G-structures $P(B)$ et $P\left(B^{\prime}\right)$. Alors, $\Phi^{\prime} \circ(\widetilde{\psi \wedge \phi})$ 
$=\tilde{\phi} \circ \Phi . \quad$ En conséquence, $\phi=\phi(f, \psi)$ est un isomorphisme (local) de $B$ dans $B^{\prime}$.

La deuxième assertion exige que l'application $\Phi: P(B) \rightarrow B$ soit surjective. Mais il est facile de trouver ce fait.

Réciproquement, supposons que $F$ soit un difféomorphisme local de $M(G)$ dans $M^{\prime}\left(G^{\prime}\right)$ tel que $\tilde{F}\left(\left.P(B)\right|_{U_{F}}\right)=\left.P\left(B^{\prime}\right)\right|_{V_{F}}$. Pour tout $A \in \mathbb{B S}_{0}^{*}$ et tout $X \in P(B)$ avec $\varpi(X)=s \in U_{F}$, on a

$$
F_{*}\left(A_{s}^{*}\right)=F_{*}(X(A))=(\tilde{F}(X))(A)=A_{F(s)}^{*} .
$$

Ceci signifie que $F$ transporte les fibres par rapport à $\mu$ dans les fibres par rapport à $\mu^{\prime}$; il existe, de cela, un difféomorphisme $\phi$ de $M$ dans $M^{\prime}$ tel que

$$
\mu^{\prime} \circ F=\phi \circ \mu \text {. }
$$

En suite, soit $a(t)(0 \leqq t \leqq 1)$ une courbe différentiable de $U_{F} \subset M(G)$ vérifiant $\lambda_{\circ}(a(t))=\lambda_{\circ}(a(0))$; par suite, $\lambda_{*} \circ(\dot{a}(t))=0$, où $\dot{a}(t)$ désigne la dérivée de $a(t)$ en $t$. Comme $\dot{a}(t)$ appartient à $T_{a(t)}^{0}(\lambda)$, d'après le Lemme 2-3, il existe un élément $w_{t} \in W$ et un élément $X_{t} \in P(B)$ avec $\widetilde{\varpi}\left(X_{t}\right) \in U_{F}$ tels que $X_{t}\left(w_{t}\right)=\dot{a}(t)$. On a donc $\left(\tilde{F}\left(X_{t}\right)\right)\left(w_{t}\right)=F_{*}(\dot{a}(t))$ et $\lambda_{*}^{\prime} \circ F_{*} \circ(\dot{a}(t))$ $=0$ car $F$ est un isomorphisme local de $P(B)$ dans $P\left(B^{\prime}\right)$. Ceci signifie que $F$ entraîne un difféomorphisme local $\tilde{\psi}$ de $\Pi(\mathscr{V}, G)$ dans $\Pi\left(\mathscr{V}^{\prime}, G^{\prime}\right)$ tel que

$$
\lambda^{\prime} \circ \boldsymbol{F}=\tilde{\psi} \circ \lambda
$$

En vertu de (2-1), on a, pour tout $A \in \mathbb{S}_{0}^{*}$,

$$
\tilde{\psi}_{*}\left(A^{*}\right)=\tilde{\psi}_{*}\left(\lambda_{*}\left(A^{*}\right)\right)=\lambda_{*}^{\prime} \circ F_{*}\left(A^{*}\right)=\lambda_{*}^{\prime}\left(A^{*}\right)=A^{*} .
$$

En conséquence, on obtient

$$
\tilde{\psi}(u \cdot \exp t A)=\tilde{\psi}(u) \cdot \exp t A
$$

pour tout $u \in U_{\tilde{\psi}}$, tout $A \in \mho_{0}^{*}$ et $t \in \boldsymbol{R}$ suffisamment petit. Ceci démontre qu'il existe un difféomorphisme local $f$ de $N$ dans $N^{\prime}$ tel que $a^{\prime} \circ \tilde{\psi}=f \circ a$ $(a: \Pi(\mathscr{V}, G) \rightarrow N$, etc.).

Soient $u, u^{\prime} \in U_{\tilde{\psi}}$ et $v, v^{\prime} \in \boldsymbol{R}^{n}$ tels que $u(v)=u^{\prime}\left(v^{\prime}\right)$; alors il existe un élément $g \in G_{0}^{*}$ vérifiant $u^{\prime}=u \cdot g$ et $v=g\left(v^{\prime}\right)$. Comme $G_{0}^{*}$ est connexe, on a, de la relation $(2-4), \tilde{\psi}(u)(v)=\tilde{\psi}\left(u^{\prime}\right)\left(v^{\prime}\right)$. Donc on peut définir un isomorphisme local de fibrés vectoriels $\psi: \mathscr{V} \rightarrow \mathscr{V}^{\prime}$ au-dessus de $f$ par 


$$
\psi(u(v))=\tilde{\psi}(u)(v) \quad(\text { ou } \psi \circ u=\tilde{\psi}(u))
$$

pour tout $u \in U_{\tilde{\psi}}$ et tout $v \in \boldsymbol{R}^{n}$.

En considérant les applications $\phi, \tilde{\psi}$ et $f$ obtenues autour du même point $\left(u_{0}, x_{0}\right) \in U_{F} \subset M(G)$, on a facilement $\alpha^{\prime} \circ \phi \circ \mu=f \circ \alpha \circ \mu$ et par suite $\alpha^{\prime} \circ \phi=f \circ \alpha$. Les relations (2-2), (2-3) et (2-5) signifient que $F$ coïncide avec $\psi \wedge \phi$ autour de point $\left(u_{0}, x_{0}\right) \in U_{F}$. Comme $F=\psi \wedge \phi$ est un isomorphisme local des deux structures $P(B)$ et $P\left(B^{\prime}\right)$, d'après le Lemme 2-6, il en résulte que $\phi=\phi(f, \psi)$ est un isomorphisme local de $B$ dans $B^{\prime}$.

Soit $B(M, \alpha, N, \mathscr{V})$ une $G \cdot G$-structure de groupe structural $G$ et de type $\left(V, W, G_{0}\right)$ et soit $\mathbb{S}_{0}$ l'algébre de Lie de $G_{0}$. En posant $V^{\prime}=\mathbb{S}_{0}^{*} \oplus V$, nous définissons une application linéaire $\partial$ de $\operatorname{Hom}\left(V^{\prime}, \oiint_{0}^{\#}\right)$ dans Hom $\left(V^{\prime} \wedge V^{\prime}, V^{\prime}\right)$;

$$
(\partial S)(X \wedge Y)=S(X)(Y)-S(Y)(X)
$$

pour tout $S \in \operatorname{Hom}\left(V^{\prime}, \mathbb{\mho}_{0}^{\sharp}\right)$ et tous $X, Y \in V^{\prime}$, et posons

$$
H^{0,2}=H^{0}{ }^{2}\left(V, W, G_{0}\right)=\operatorname{Hom}\left(V^{\prime} \wedge V^{\prime}, V^{\prime}\right) / \operatorname{Im} \partial .
$$

D'après la thèorie générale, il existe l'application $C_{B}: P(B) \rightarrow H^{0,2}$, la fonction structurale de $P(B)$ (voir [12]). Prenons une connexion $\Omega$ sur l'espace fibré principal $P(B)$. En désignant par $\omega$ la forme extérieure canonique sur $P(B)$ et par $\Theta$ la forme de torsion, la première equation structurale est représentée par

$$
d \omega=-\Omega \wedge \omega+\Theta .
$$

Soit $\left\{\boldsymbol{a}_{1}, \cdots, \boldsymbol{a}_{N}\right\}$ une base de $V^{\prime}=\mathbb{S}_{0}^{*} \oplus V$, et posons $\omega=\sum_{i=1}^{N} \omega^{i} \boldsymbol{a}_{i} \Theta=$ $\sum_{i=1}^{N} \Theta^{i} \boldsymbol{a}_{i}$. On peut vérifier l'equation

$$
\Theta^{i}=\frac{1}{2} \sum_{j, k=1}^{N} C_{j k}^{i} \omega^{j} \wedge \omega^{k} \quad(i=1,2, \cdots, N),
$$

où $C_{j k}^{i}$ sont des fonctions différentiables sur $P(B)$ (cf. [7]). Définissons l'élément $C \in \operatorname{Hom}\left(V^{\prime} \wedge V^{\prime}, V^{\prime}\right)$ par

$$
C\left(\boldsymbol{a}_{i} \wedge \boldsymbol{a}_{j}\right)=\sum_{k=1}^{N} C_{i j}^{k} \cdot \boldsymbol{a}_{k}
$$

Il se trouve que l'image $\bar{C} \in H^{0,2}$ de $C$ coïncide, au fond, avec la fonction structurale $C_{B}$ de $P(B)$.

Soit $\theta$ la forme canonique sur $B$ à valeurs dans $\mathscr{V}$ et soit $\Phi: P(B)$ 
$\rightarrow B$ l'application définie dans la Proposition 2-2. Alors on aura

$$
\lambda(s)^{-1}\left(\Phi^{*} \theta\right)_{X}(Z)=V \text {-composant de } \omega_{X}(Z)
$$

$\left(X \in P(B), s=\widetilde{\varpi}(X), Z \in T_{X}(P(B))\right)$. Si $\mathscr{V}$ est trivial, i.e., $\mathscr{V}=N \times V$, on peut définir la différentielle extérieure $d \theta$ de $\theta$ et, à l'aide de $d \theta$, on pourra construire la fonction structurale de $B$. Mais la relation entre les deux fonctions structurales sera assez compliquée.

\section{§3. Le cas analytique}

Dans ce paragraphe, tous les objets sont supposés analytiques. Tout ce que nous avons obtenu jusqu'à présent est encore vrai en remplaçant le mot "différentiable" par "analytique réel". Nous nous proposons dans ce cas-là d'indiquer certain théorème qui ramène partiellement le problème d'équivalence des $G \cdot G$-structures au problème entièrement algébrique.

Soient $V, W$ des espaces vectoriels réels de dimension finie, et $E$ un sous-espace vectoriel de $\operatorname{Hom}(V, W)$. Nous définissons le prolongement algébrique d'ordre 1 de $E$ par

$$
E^{(1)}=\{X \in \operatorname{Hom}(V, E) \mid X(u)(v)=X(v)(u), u, v \in V\},
$$

et posons, pour une base $(v)=\left\{v_{1}, \cdots, v_{n}\right\}$ de $V(n=\operatorname{dim} V)$,

$$
E_{k}(v)=\left\{X \in E \mid X\left(v_{1}\right)=\cdots=X\left(v_{k}\right)=0\right\} \quad(k=1,2, \cdots, n) .
$$

Definition 3-1. $E \subset \operatorname{Hom}(V, W)$ est dit involutif, si et seulement s'il existe une base $(v)=\left\{v_{1}, \cdots, v_{n}\right\}$ de $V$ telle que

$$
\operatorname{dim} E^{(1)}=\operatorname{dim} E+\sum_{k=1}^{n} \operatorname{dim} E_{k}(v) .
$$

En général, on a toujours

$$
\operatorname{dim} E^{(1)} \leqq \operatorname{dim} E+\sum_{k=1}^{n} \operatorname{dim} E_{k}(v)
$$

quelle que soit la base $(v)=\left\{v_{1}, \cdots, v_{n}\right\}$ de $V$ (cf. [12]).

Soient $\left(V, W, G_{0}\right)$ un système compatible, $B=B(M, \alpha, N, \mathscr{V}$ ) (resp. $\left.B^{\prime}=B^{\prime}\left(M^{\prime}, \alpha^{\prime}, N^{\prime}, \mathscr{V}^{\prime}\right)\right)$ une $G \cdot G$-structure de type $\left(V, W, G_{0}\right)$ et de groupe structural $G$ (resp. $\left.G^{\prime}\right), P(B)$ (resp. $P\left(B^{\prime}\right)$ ) la $G_{0}^{\#}$-structure associée à $B$ (resp. $B^{\prime}$ ) et $\omega$ (resp. $\omega^{\prime}$ ) la forme extérieure canonique sur $P(B)$ (resp. $P\left(B^{\prime}\right)$ ) à valeurs dans $\mathbb{S}_{0}^{*} \oplus V$. En désignant par $P_{1}$ (resp. $P_{2}$ ) la projection canonique de $P(B) \times P\left(B^{\prime}\right)$ sur $P(B)$ (resp. $P\left(B^{\prime}\right)$ ), nous définissons 
une forme extérieure $\Omega$ sur $P(B) \times P\left(B^{\prime}\right)$ par la formule;

$$
\Omega=P_{1}^{*} \omega-P_{2}^{*} \omega^{\prime} \text {. }
$$

Alors $\left\{\Omega, P_{1}^{*} \omega\right\}$ engendre un système différentiel extérieur avec des variables independantes ([6], [9]). Ce système est en involution, si l'algèbre de Lie de $G_{0}^{\#}$, 厄্: $C_{B^{\prime}}$ soient constantes et $C_{B}=C_{B^{\prime}}$ (cf. [6], [12]). Par conséquent, on peut retrouver le théorème suivant en utilisant le Théorème de Cartan-Kähler (cf. [9]).

THEOREME 3-1. Si les conditions suivantes sont vérifiées;

i) ङS\# est involutif,

ii) les fonctions $C_{B}$ et $C_{B^{\prime}}$ sont constantes, et $C_{B}=C_{B^{\prime}}$, alors $P(B)$ et $P\left(B^{\prime}\right)$ sont localement isomorphes. Autrement dit, pour tout $X \in P(B)$ et tout $X^{\prime} \in P\left(B^{\prime}\right)$, il existe un difféomorphisme local $\phi$ d'un voisinage de $\widetilde{\sigma}(X)$ sur celui de $\widetilde{\sigma}^{\prime}\left(X^{\prime}\right)$ tel que $\tilde{\phi}\left(\left.P(B)\right|_{U_{\phi}}\right)=\left.P\left(B^{\prime}\right)\right|_{V_{\phi}}$, $\tilde{\phi}(X)=X^{\prime}$.

Au point de vue des $G \cdot G$-structures $B$ et $B^{\prime}$, les conditions i) et ii) de ce théorème seront indirectes. Mais, en ce qui concerne la condition i), on peut établir le théorème suivant.

THEOREME 3-2. Soit (V,W, (s) un système compatible. Pour que l'algéble (3) soit involutive, il faut et il suffit que (3) soit involutif.

Laissons la démonstration de ce théorème pour le moment.

Theoreme 3-3. Soit $B$ (resp. $B^{\prime}$ ) une G.G-structure analytique réel de base $(M, \alpha, N, \mathscr{V})$ (resp. $\left.\left(M^{\prime}, \alpha^{\prime}, N^{\prime}, \mathscr{V}^{\prime}\right)\right)$ et de type $\left(V, W, G_{0}\right)$. Si les conditions suivantes sont vérifiées;

i) $\mathbb{G}_{0}$ est involutif,

ii) $C_{B}$ et $C_{B^{\prime}}$ sont constants et $C_{B}=C_{B^{\prime}}$,

alors $B$ et $B^{\prime}$ sont localement isomorphes. En conséquence, les pseudogroupes $\Gamma(B)$ et $\Gamma\left(B^{\prime}\right)$ sont localement isomorphes.

Ce théorème résulte du Théorème 2-2, du Théorème 3-1 et du Théorème 3 -2.

THEOREME 3-4. Soit $B(M, \alpha, N, \mathscr{V})$ une $G \cdot G$-structure analytique réel de type $\left(V, W, G_{0}\right)$. Supposons que $\mathbb{S}_{0}$ soit involutif et que $C_{B}$ soit constant sur $P(B)$. Alors le pseudo-groupe des automorphismes de $B, \Gamma^{*}(B)$, 
opère transitivement sur $M$. En conséquence, le normalisateur $N(\Gamma)$ de $\Gamma=\Gamma(B)$ est transitif sur $M$, et les pseudo-groupes $\Gamma(B)_{\xi}(\xi \in N)$ restreints aux fibres de la fibration $\alpha: M \rightarrow N$ sont aussi localement isomorphes.

Ce théorème résulte $d u$ Théorème 2-3, du Corollaire 2-1 et du Théorème 3-3.

Voici maintenant trouvons-nous le Théorème 3-2. Soit $(V, W$, (6) un système compatible. En posant $\mathfrak{S}=\operatorname{Hom}(V / W$, (S) $)$, on a

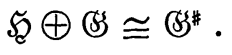

L’isomorphisme est donné par

$$
\phi(X, Y)(A, v)=(X(\pi(v)), Y(v))
$$

( $X \in \mathfrak{S}, Y \in \mathbb{B}, A \in \mathbb{S}^{*}, v \in V$ ), où $\pi$ désigne l'application canonique $V \rightarrow V / W$. Soient $n$ la dimension de $V$ et $m$ la dimension de $V / W$. Posons, pour une base $(x)=\left\{x_{1}, \cdots, x_{n}\right\}$ de $V$,

$$
\begin{aligned}
& \widetilde{\mathbb{S}}_{k}^{\sharp}(x)=\left\{X \in \mathbb{S}^{\#} \mid X\left(x_{1}\right)=\cdots=X\left(x_{k}\right)=0\right\}, \\
& \widetilde{\mathfrak{S}}_{k}(x)=\left\{Y \in \mathfrak{S}^{\prime} \mid Y\left(\pi\left(x_{1}\right)\right)=\cdots=Y\left(\pi\left(x_{k}\right)\right)=0\right\}, \\
& \mathbb{S}_{k}(x)=\left\{Z \in \mathbb{S} \mid Z\left(x_{1}\right)=\cdots=Z\left(x_{k}\right)=0\right\},
\end{aligned}
$$

$(k=1,2, \cdots, n)$. En vertu de (3-1), on a aisément le

LEMME 3-1. Pour tout $k=1,2, \cdots, n$,

$$
\widetilde{\mathbb{S}}_{k}^{\sharp}(x) \cong \widetilde{\mathfrak{S}}_{k}(x) \oplus \mathbb{S}_{k}(x) \text {. }
$$

Ensuite, posons

$$
p(\mathfrak{S})=\{\tilde{H} \in \operatorname{Hom}(V, \mathscr{S}) \mid \tilde{H}(u)(\pi(v))=\tilde{H}(v)(\pi(u)), u, v \in V\} .
$$

Soient $\tilde{H} \in p(\mathfrak{S})$ et $w \in W$. Pour tout $u \in V$, on a $\tilde{H}(w)(\pi(u))=\tilde{H}(u)$ $\cdot(\pi(w))=0$, par suite, $\tilde{H}(w)=0$ quel que soit $w$ dans $W$. Donc $p(\mathfrak{S})$ s'identifie à l'espace vectoriel

$$
\mathfrak{S E}^{(1)}=\{H \in \operatorname{Hom}(V / W, \mathfrak{S}) \mid H(\xi)(\eta)=H(\eta)(\xi), \xi, \eta \in V / W\} .
$$

Par conséquent, on a

$$
\operatorname{dim} p(\mathscr{S})=\operatorname{dim} \mathscr{S}^{(1)}=\operatorname{dim}()^{*} \times \frac{m(m+1)}{2} .
$$


Posons, dé plus,

$$
\mathscr{S}_{k}(z)=\left\{Y \in \mathfrak{S}_{\mathcal{C}} \mid Y\left(z_{1}\right)=\cdots=Y\left(z_{k}\right)=0\right\} \quad(k=1,2, \cdots, m),
$$

pour une base $(z)=\left\{z_{1}, \cdots, z_{m}\right\}$ de $V / W$. Alors on obtient le

Lemme 3-2. Pour toute base (z) de $V / W$,

$$
\operatorname{dim} p(\mathfrak{S})=\operatorname{dim} \mathfrak{S}_{\mathfrak{C}}+\sum_{k=1}^{m-1} \operatorname{dim} \mathfrak{S}_{k}(z) .
$$

En effet, posons $\tilde{V}=V / W$ et désignons par $W_{k}(z)$ le sous-espace vectoriel de $\tilde{V}$ engendré par $z_{1}, \cdots, z_{k}(k=1,2, \cdots, m)$. Il est facile de démontrer que $\mathfrak{S}_{k}(z)$ s'identifie à l'espace vectoriel $\operatorname{Hom}\left(\tilde{V} / W_{k}(z)\right.$, \&s*). Alors on a $\operatorname{dim} \mathscr{S}_{k}(z)=\operatorname{dim} \mathfrak{s}^{*} \times(m-k)$, et par suite,

$$
\sum_{k=1}^{m-1} \operatorname{dim} \mathscr{S}_{k}(z)=\operatorname{dim}()^{*} \times \sum_{k=1}^{m-1}(m-k)=\operatorname{dim} \oiint^{*} \times \frac{m(m-1)}{2} .
$$

Et l'assertion résulte de la relation $\operatorname{dim} \mathfrak{S}=\operatorname{dim}(5) \times m$ et $(3-2)$.

LEMME 3-3. $\quad(\text { (S) })^{(1)} \cong p(\mathfrak{S}) \oplus \mathbb{S}^{(1)}$.

En effet, l'espace vectoriel Hom (\&্* $\oplus V$, (S) s'identifie à la somme

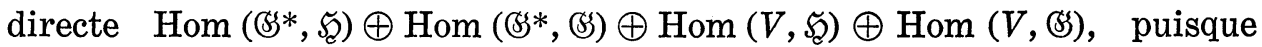
(ङ) $\cong \mathscr{S} \oplus$ (S). Il en résulte que $X \in\left(\mathbb{S}^{\#}\right)^{(1)}$ peut être partagé en quatre, i.e., $X=X_{1}+X_{2}+X_{3}+X_{4}, X_{1} \in \operatorname{Hom}(\mathbb{S} *, \mathfrak{S}), X_{2} \in \operatorname{Hom}\left(\mathbb{S} *\right.$, (S)), $X_{3} \in \operatorname{Hom}(V, \mathfrak{S})$ et $X_{4} \in \operatorname{Hom}(V, \mathbb{S})$. Comme $X$ satisfait à la relation $X(A, u)(B, v)=$ $X(B, v)(A, u)$ pour tous $A, B \in \mathbb{S} *$ et tous $u, v \in V$, on a

$$
\begin{gathered}
X_{1}(A)(\pi(v))=X_{1}(B)(\pi(u)) \\
X_{2}(A)(v)=X_{2}(B)(u) \\
X_{3}(u)(\pi(v))=X_{3}(v)(\pi(u)) \\
X_{4}(u)(v)=X_{4}(v)(u) .
\end{gathered}
$$

Si $B=0$ dans les relations (3-3) et (3-4), on a $X_{1}=X_{2}=0$. Par suite $\left(\mathbb{( s}^{\sharp}\right)^{(1)} \subset \operatorname{Hom}(V, \mathfrak{S}) \oplus \operatorname{Hom}(V$, (s) $) . \quad$ Mais (3-5) et (3-6) signifient que $X_{3} \in p(\mathfrak{S})$ et $X_{4} \in \mathscr{S S}^{(1)}$.

Démonstration du Théorème 3-2. Supposons tout d'abord que đ্s soit involutif. Soit $(x)=\left\{x_{1}, \cdots, x_{n}\right\}$ une base de $V$ vérifiant la relation $\operatorname{dim} \mathscr{S}^{(1)}=\operatorname{dim} \mathbb{S}+\sum_{k=1}^{n-1} \operatorname{dim} \mathbb{S}_{k}(x)$. Nous pouvons supposer que $\left\{\pi\left(x_{1}\right)\right.$, $\left.\cdots, \pi\left(x_{m}\right)\right\}$ soit une base de $V / W$ en remplaçant la base $(x)$, s'il en est 
besoin.

Ceci étant, on a facilement

$$
\begin{cases}\widetilde{\mathfrak{S}}_{k}(x)=\mathfrak{S}_{k}(z) & (k=1,2, \cdots, m), \\ \widetilde{\mathfrak{S}}_{k}(x)=0 & (k=m, m+1, \cdots, n),\end{cases}
$$

en posant $(z)=\left\{\pi\left(x_{1}\right), \cdots, \pi\left(x_{m}\right)\right\}$. Prenons une base de $\mathbb{S}^{*} *,\left\{x_{n-1}, \cdots, x_{N}\right\}$; $(\bar{x})=\left\{x_{1}, \cdots, x_{n}, x_{n+1}, \cdots, x_{N}\right\}$ devient une base de $\&^{*} \oplus V$. D'après la définition de 졔, on a

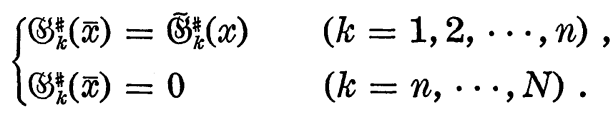

Les Lemmes 3-2,3-3 entraînent

$$
\begin{aligned}
\operatorname{dim}\left(\mathscr{S}^{\sharp}\right)^{(1)} & =\operatorname{dim} p(\mathscr{S})+\operatorname{dim} \mathscr{S S}^{(1)} \\
& =\operatorname{dim} \mathfrak{S}_{\mathcal{C}}+\sum_{k=1}^{m-1} \operatorname{dim} \mathfrak{S}_{k}(z)+\operatorname{dim} \mathbb{S}+\sum_{k=1}^{n-1} \operatorname{dim} \mathbb{S}_{k}(x) \\
& =\operatorname{dim}\left(\mathfrak{S C}_{\mathfrak{S}} \oplus \mathbb{S}\right)+\sum_{k=1}^{n-1} \operatorname{dim}\left(\mathfrak{S}_{k}(z) \oplus \mathbb{S}_{k}(x)\right) .
\end{aligned}
$$

En vertu de la relation $\mathscr{C S}^{\#} \cong \mathscr{S} \oplus \mathbb{S}$ et du Lemme 3-1 et des (3-7), (3-8),

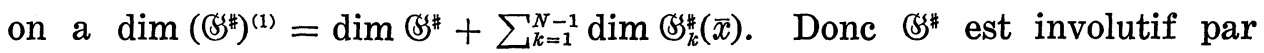
rapport à la base $(\bar{x})$.

Inversement, supposons que $\mathbb{S}^{\sharp}$ soit involutif. Soit $(v)=\left\{v_{1}, v_{2}, \cdots\right.$,

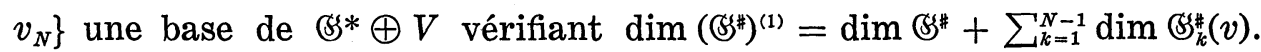
Posons $v_{i}=A_{i}+x_{i}\left(A_{i} \in \mathbb{S}^{*}, x_{i} \in V, i=1,2, \cdots, N\right)$. Comme $x_{1}, \cdots, x_{N}$ engendrent l'espace vectoriel $V$, on peut choisir une base $\left\{x_{k_{1}}, \cdots, x_{k_{n}}\right\}$ de $V$ telle que

i) $\left\{x_{k_{1}}, \cdots, x_{k_{n}}\right\} \subset\left\{x_{1}, \cdots, x_{N}\right\}$ et $1 \leqq k_{1}<k_{2}<\cdots<k_{n} \leqq N$,

ii) $x_{1}=\cdots=x_{k_{1-1}}=0$, si $k_{1} \geqq 2$,

iii) pour tout $i \geqq 1, x_{k_{i}}, x_{k_{i+1}}, \cdots, x_{k_{i+1}-1}$ sont représentés par la combination linéaire des $x_{k_{1}}, \cdots, x_{k_{i}}$. Posons $y_{i}=x_{k_{i}}(i=1,2, \cdots, n)$. Comme (s) annule (s)*, on a facilement

a) $\mathbb{S}_{1}^{\#}(v)=\cdots=\mathbb{S}_{k_{1}-1}^{\#}(v)=\mathbb{S}_{\#}^{\#}$ si $k_{1} \geqq 2$,

b) $\widetilde{\mathbb{S}}_{i}^{\sharp}(y)=\mathbb{S}_{j}^{\sharp}(v) \quad\left(j=k_{i}, k_{i}+1, \cdots, k_{i+1}-1, i=1,2, \cdots, n\right)$,

c) $\mathbb{S}_{i}^{\sharp}(v)=0 \quad\left(i=k_{n}, k_{n}+1, \cdots, N\right)$,

et par suite,

$$
\begin{aligned}
& \sum_{i=1}^{N-1} \operatorname{dim} \mathbb{S}_{i}^{\sharp}(v)=\sum_{i=1}^{k_{1}-1} \operatorname{dim} \mathbb{S}_{i}^{\#}(v)+\sum_{\ell=1}^{n-1}\left(\sum_{\substack{k \ell k_{\ell} \\
i \ell+1}}^{N+1} \operatorname{dim} \mathbb{S}_{i}^{\#}(v)\right) \\
& +\sum_{i=k_{n}}^{N-1} \operatorname{dim} \mathscr{S}_{i}^{\#}(v) \\
& =\left(k_{1}-1\right) \operatorname{dim} \mathbb{S}^{\#}+\sum_{i=1}^{n-1}\left(k_{i+1}-k_{i}\right) \operatorname{dim} \widetilde{\Im}_{i}^{\#}(y) \text {. }
\end{aligned}
$$


On obtient, en outre,

$$
\sum_{i=1}^{N-1} \operatorname{dim} \mathcal{S}_{i}^{\sharp}(v) \geqq \sum_{j=1}^{n-1} \operatorname{dim} \widetilde{\Im}_{j}^{\sharp}(y),
$$

puisque $k_{1}-1 \geqq 0$ et $k_{i+1}-k_{i} \geqq 1$ pour tout $i=1,2, \cdots, n-1$. D'après le Lemme $3-1$, le Lemme $3-3$ et (3-9), on a

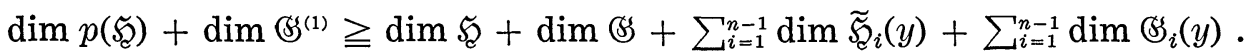
$\pi\left(y_{1}\right), \cdots, \pi\left(y_{n}\right)$ engendrent l'espace vectoriel $V / W$, c'est pour cela que

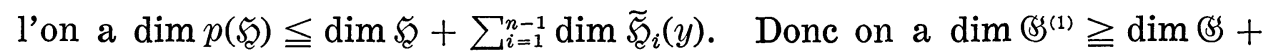
$\sum_{i=1}^{n-1} \operatorname{dim} \oiint_{i}(y)$. Ceci signifie que \&S est involutif.

c.q.f.d.

\section{§4. Exemple}

Nous allons calculer un exemple qui a été pris par E. Cartan ([2] $n^{\circ}$ 29). Il s'agit d'un exemple très simple qui pourtant contient en soi les subtilités de la théorie.

Soient $M=\boldsymbol{R}^{2}$ muni des coordonnées $(x, y)$ et $N=\boldsymbol{R}$ muni de $x$. Considérons la fibration évidente $\alpha: M \rightarrow N,(x, y) \mapsto x$, et considérons le pseudo-groupe sur $M$

$$
\Gamma:\left\{\begin{array}{l}
X=x \\
Y=y+f(x),
\end{array}\right.
$$

où $f(x)$ désigne une fonction locale différentiable.

Soit $V=\boldsymbol{R}^{2}$ muni de la base $\left\{\boldsymbol{e}_{1}, \boldsymbol{e}_{2}\right\}$ canonique, et désignons par $W$ le sous-espace vectoriel de $V$ engendré par l'élément $e_{2}$. Considérons, de plus, le groupe de Lie $G_{0}$ de $G L(n, R)$ formé par les éléments sous la forme

$$
\left(\begin{array}{ll}
1 & 0 \\
p & 1
\end{array}\right) \in G L(2, \boldsymbol{R}), \quad p \in \boldsymbol{R}
$$

Alors $\left(V, W, G_{0}\right)$ est un système compatible (cf. §2).

Définissons une section $I: N \rightarrow M$ par $I(x)=(x, 0), x \in N$. Comme $\mathscr{V}=I^{*} T(M)$ est trivial, la $G \cdot G$-structure $B$ déterminée par $\Gamma$ est considérée comme une sous-variété différentiable de l'espace fibré des repères de $M, F(M)$. Considérons la section différentiable de $F(M) ; \tau(x, y)\left(e_{1}\right)=$ $\left(\frac{\partial}{\partial x}\right)_{(x, y)}, \tau(x, y)\left(e_{2}\right)=\left(\frac{\partial}{\partial y}\right)_{(x, y)},(x, y) \in M$. Il se trouve que la $G \cdot G$-structure $B$ est isomorphe à $M \times G_{0}$; l'isomorphisme est donné par $\phi: M \times G_{0} \rightarrow B$, 
$\phi((x, y), g)=\tau(x, y) g,(x, y) \in M, g \in G_{0}$. Il est immédiat de constater que $G_{0}^{*}$ est formé par les éléments sous la forme

$$
\left(\begin{array}{ll}
a & 0 \\
b & c
\end{array}\right) \in G L(2, \boldsymbol{R}), \quad a, b, c \in \boldsymbol{R}, \quad a c \neq 0 .
$$

Comme le groupe structural de $B$ coïncide avec $G=N \times G_{0}, \Pi(\mathscr{V}, G)$ s'identifie à $N \times G_{0}^{*}$. Donc $B$ est de type $\left(V, W, G_{0}\right)$. On a aussi $M(G)$ $\cong M \times G_{0}^{*}$, puisque $\Pi(\mathscr{V}, G) \cong N \times G_{0}^{*}$ (cf. Proposition 2-1).

Soit $\oiint_{0}^{*}$ l'algébre de Lie de $G_{0}^{*}$, i.e.,

$$
\mathbb{S}_{0}^{*}=\left\{\left(\begin{array}{ll}
a & 0 \\
b & c
\end{array}\right) \in \mathfrak{g} \mathfrak{l}(2, \boldsymbol{R}) \mid a, b, c \in \boldsymbol{R}\right\} .
$$

Prenons la base $\left\{X_{1}, X_{2}, X_{3}\right\}$ de $\mho ্ S_{0}^{*}$;

$$
X_{1}=\left(\begin{array}{ll}
1 & 0 \\
0 & 0
\end{array}\right), \quad X_{2}=\left(\begin{array}{ll}
0 & 0 \\
1 & 0
\end{array}\right), \quad X_{3}=\left(\begin{array}{ll}
0 & 0 \\
0 & 1
\end{array}\right)
$$

vérifiant les relations

$$
\left[X_{1}, X_{2}\right]=-X_{2}, \quad\left[X_{1}, X_{3}\right]=0, \quad\left[X_{2}, X_{3}\right]=-X_{2} .
$$

Calculons les champs de vecteurs $X_{i}^{*}$ sur $M(G), i=1,2,3$, on a

$$
X_{1}^{*}=a\left(\frac{\partial}{\partial a}\right)+b\left(\frac{\partial}{\partial b}\right), \quad X_{2}^{*}=c\left(\frac{\partial}{\partial b}\right), \quad X_{3}^{*}=c\left(\frac{\partial}{\partial c}\right),
$$

où $(a, b, c, x, y)$ désigne le système des coordonnées de $M(G)$. En suivant la construction dans la démonstration du Lemme 2-2, nous construisons la section différentiable de $\varpi: P(B) \rightarrow M(G)$ par rapport à la section $\tau: M \rightarrow B$; on a aisément

$$
\begin{aligned}
& \tilde{\tau}(u)(A)=A_{u}^{*} \quad A \in \mathbb{\mho}_{0}^{*}, \\
& \tilde{\tau}(u)\left(\boldsymbol{e}_{1}\right)=a\left(\frac{\partial}{\partial x}\right)_{u}+b\left(\frac{\partial}{\partial y}\right)_{u}, \\
& \tilde{\tau}(u)\left(\boldsymbol{e}_{2}\right)=c\left(\frac{\partial}{\partial y}\right)_{u},
\end{aligned}
$$

pour tout $u=(a, b, c, x, y) \in M(G)$.

Il est immédiat de constater que $G_{0}^{\#}$ est formé par les éléments sous la forme 


$$
\left[\begin{array}{lllll}
1 & 0 & 0 & \alpha & 0 \\
0 & 1 & 0 & \beta & 0 \\
0 & 0 & 1 & \gamma & 0 \\
0 & 0 & 0 & 1 & 0 \\
0 & 0 & 0 & p & 1
\end{array}\right] \in G L(5, \boldsymbol{R}), \quad \alpha, \beta, \gamma, p \in \boldsymbol{R}
$$

Donc $(a, b, c, x, y, \alpha, \beta, \gamma, p)$ est considéré comme un système de coordonnées de $P(B)$.

Soit $\omega$ la forme extérieure canonique sur $P(B)$ à valeurs dans $\oiint^{*} \oplus V$. En posant $\omega=\omega^{1} \cdot X_{1}+\omega^{2} \cdot X_{2} \cdot+\omega^{3} \cdot X_{3}+\omega^{4} \cdot e_{1}+\omega^{5} \cdot e_{2}$, calculons chaque $\omega^{i}$; on a

$$
\begin{aligned}
& \omega^{1}=-\frac{\alpha}{a} d x+\frac{d a}{a}, \\
& \omega^{2}=-\frac{\beta}{a} d x-\frac{b}{c} \frac{d a}{a}+\frac{1}{c} d b, \\
& \omega^{3}=-\frac{\gamma}{a} d x+\frac{d c}{c}, \\
& \omega^{4}=\frac{1}{a} d x, \\
& \omega^{5}=-\frac{b+p c}{a c} d x+\frac{1}{c} d y,
\end{aligned}
$$

et par suite,

$$
\begin{aligned}
& d \omega^{1}=-\Omega^{1} \wedge \omega^{4}, \\
& d \omega^{2}=\omega^{1} \wedge \omega^{2}+\omega^{2} \wedge \omega^{3}-\Omega^{2} \wedge \omega^{4}, \\
& d \omega^{3}=-\Omega^{3} \wedge \omega^{4}, \\
& d \omega^{4}=-\omega^{1} \wedge \omega^{4}, \\
& d \omega^{5}=-\omega^{2} \wedge \omega^{4}-\omega^{3} \wedge \omega^{5}-\Omega^{4} \wedge \omega^{4},
\end{aligned}
$$

où $\Omega^{1}, \cdots, \Omega^{4}$ désignent respectivement les formes

$$
\begin{aligned}
& \Omega^{1}=d \alpha-\alpha \omega^{1}, \\
& \Omega^{2}=d \beta-2 \beta \omega^{1}+\beta \omega^{3}+(\alpha-\gamma) \omega^{2}, \\
& \Omega^{3}=d \gamma-\gamma \omega^{1}, \\
& \Omega^{4}=d p-p \omega^{1}+p \omega^{3}-\gamma \omega^{5} .
\end{aligned}
$$

Il en résulte que la fonction structurale $C_{B}$ de $P(B)$ est constante.

Soit $F$ un isomorphisme locale de $P(B)$. En posant $a \circ F=F_{1}, b \circ F$ 
$=F_{2}, c \circ F=F_{3}, x \circ F=F_{4}$ et $y \circ F=F_{5}$, calculons chaque $F_{i}$; on a

$$
\begin{aligned}
& F_{1}=f^{\prime}(x) a, \\
& F_{2}=g(x) b+A(x) c, \\
& F_{3}=g(x) c, \\
& F_{4}=f(x), \\
& F_{5}=g(x) y+h(x),
\end{aligned}
$$

où $f, g, h$ et $A$ désignent des fonctions locales différentiables et $f^{\prime}$ la dérivée de $f$. D'après la théorie générale, le pseudo-groupe qui laisse $\Gamma$ invariant est donné par

$$
\Gamma^{*}: \phi=\left\{\begin{array}{l}
X=f(x) \\
Y=g(x) y+h(x)
\end{array}\right.
$$

Dans ce cas-là, ce pseudo-groupe coïncide avec le normalisateur $N(\Gamma)$ de $\Gamma$. L'isomorphisme de fibrés vectoriels $\psi$ associé à l'élément $\phi$ est donné par

$$
\begin{aligned}
& \psi\left(e_{1}\right)=f^{\prime}(x) e_{1}+A(x) \boldsymbol{e}_{2}, \\
& \psi\left(e_{2}\right)=g(x) \boldsymbol{e}_{2} .
\end{aligned}
$$

En posant $\psi=$ id, on voit que l'élément $\phi$ appartient encore à $\Gamma$.

\section{BIBLIOGRAPHIE}

[1] E. Cartan, Sur la structure des groupes infinis de transformations, Ann. Ec. Norm., 21 (1904), 153-206; 22 (1905), 219-308.

[2] - Les sous-groupes des groupes continus de transformations, Ann. Ec. Norm., 25 (1908), 57-194.

[ 3 ] — Les groupes de transformations continus, infinis, simples, Ann. Ec. Norm., 26 (1909), 93-161.

[ 4 ] — Les problèmes d'équivalence, Euvres II vol. 2, 1311-1334.

[5] — L La structure des groupes infinis, Euvres II vol. 2, 1335-1384.

[6] —_, "Les systèmes différentiels extérieurs et leurs application géométriques" Paris, Hermann, 1945.

[ 7 ] S. Kobayashi and K. Nomizu, "Foundations of Differential Geometry" vol. 1, New York (1963).

[ 8 ] A. Kumpera, Invariants différentiels d'un pseudo-groupe de Lie I, J. Diff. Geometry 10 (1975), 289-345.

[ 9 ] M. Kuranishi, On E. Cartan's prolongation theorem of exterior differential system, Amer. J. Math., 79 (1957), 1-47.

[10] P. Liebermann, Sur le problème d'équivalence de certaines structures infinitésimales, Annali di Mat. pura appl., 36 (1954), 27-120.

[11] Y. Matsushima, Pseudogroupes de Lie transitifs, Séminaire Bourbaki $n^{\circ} 118$ (1955). 
[12] I. M. Singer and S. Sternberg, The infinite group of Lie and Cartan, J. Analyse Math., 15 (1965), 1-114.

[13] K. Ueno, A study on the equivalence of generalized G-structures, M. Sc. thesis, Kyoto University (1968).

Department of Mathematics

Nagoya University 